\title{
Estudos climatológicos da Reserva Florestal Ducke, Manaus, Am.
}

\author{
I. Geotemperaturas $(*)$
}

\author{
Afonso Decico 1 \\ Hugo Menezes Santos ${ }^{2}$ \\ Maria de Nazaré Góes Ribeiro ${ }^{3}$ \\ Eneas Salati ${ }^{4}$
}

\section{Resumo}

Procurando fornecer informaçōes para um estudo micrometeorológico completo da Reserva Florestal Ducke, estimaram-se alguns parâmetros térmicos do solo, com cobertura vegetal. Os dados estimados indicam que nos meses de setembro, outubro e novembro, a temperatura da superfície do solo atinge seus valores mais altos, da ordem de $46^{\circ} \mathrm{C}$, nos demais meses do ano em torno de $43,5^{\circ} \mathrm{C}$. O calor absorvido pelo solo é da ordem de $95 \mathrm{cal}$ $\mathrm{cm}^{-2}$ dia ${ }^{-1}$, correspondente a aproximadamente $22 \%$ da energia global incidente. A onda diária da temperatura se extingue numa camada superficial do solo da ordem de $60 \mathrm{~cm}$ de espessura.

\section{INTRODUÇÃo}

A existência das muitas formas de vida e particularmente da vida vegetal é ampiamente dependente de uma troca vigorosa de energia na biosfera.

Esta troca de energia se processa em parte através da interação do solo com a atmosfera. Neste particular o solo tem a função de absorver e armazenar a energia solar e posteriormente aquecer a atmosfera.

A ordem de grandeza do calor absorvido pelo solo e a espessura da camada de extinção da onda diária da temperatura são parâmetros indispensáveis a um estudo micrometeorológico completo.

Neste trabalho objetivamos fornecer os dados experimentais de temperatura do perfil do solo referente ao período $1965-1975$ da Reserva Florestal Ducke.

\section{MATERLAL E MÉtodo}

DESCRIÇÕES DA ÁREA EXPERIMENTAL

Os dados experimentais de temperatura foram obtidos na Estação Meteorológica do INPA, situada na Reserva Florestal Ducke, km 26 da Rodovia Torquato Tapajós. Suas coordenadas geográficas são as seguintes: latitude $03^{\circ} 08^{\prime} \mathrm{S}$; longitude $60^{\circ} 02^{\prime} \mathrm{W}$ e altitude $84 \mathrm{me}-$ tros.

A região é coberta por floresta tropical, mas a Estação encontra-se numa clareira em terreno plano. A vegetação nessa clareira é de um modo geral baixa, sendo que na área em que estão instalados os instrumentos a cobertura vegetal do solo é de grama rasteira, e a natureza do terreno é argi!o-silicosa.

\section{TERMÔMETRO}

As temperaturas do solo foram obtidas com geotermômetros de mercúrio em vidro marca "FUESS", cuja sensibilidade é de $0,2^{\circ} \mathrm{C}$.

\section{ASPECTOS TEÓRICOS}

A energia radiante que atinge uma área unitária plana e horizontal na unidade de tempo da superfície da terra é utilizada em diferentes processos, conforme pode ser visto no gráfico I.

\footnotetext{
(*) - Trabalho inteiramente subvencionado pelo Conselho Nacional de Desenvolvimento Científico e Tecnológico "(CNPq.).

(1) - Escola Superior de Agricultura "Luiz de Queiroz" (ESALQ), Piracicaba, SP

(2) - Universidade do Amazonas, Manaus.

(3) - Instituto Nacional de Pesquisas da Amazônia, Manaus.

(4) - Centro de Energia Nuclear da Agricultura (CENA), USP.
} 


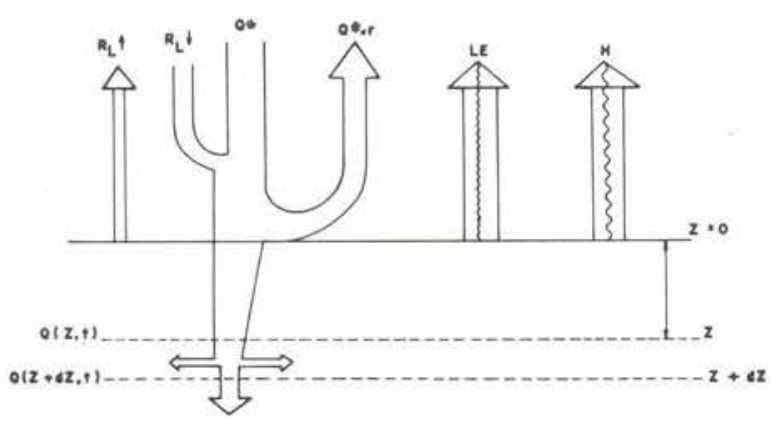

Gráfico I. Esquema do balanço de energia na superfície do solo.

onde

$Q^{*}=$ fluxo de radiação solar incidente

$Q^{*} \cdot r=$ fluxo de radiação solar refletida

$\mathbf{R}_{\mathbf{L}} \downarrow=$ fluxo de radiação de ondas longas emitidas pela atmosfera na direção da terra

$R_{L} \uparrow=$ fluxo de radiação de ondas longas emitidas pela terra

$\mathrm{LE}=$ fluxo de calor latente

$\mathrm{H}=$ fluxo de calor sensivel

Ao atingir a superfície da terra parte dessa energia solar interage com a camada superficial do solo, convertendo-se em energia térmica. A fim de se obter a equação que descreva a variabilidade e distribuição da temperatura no perfil do solo, faz-se o balanço de calor numa camada, conforme indicado no gráfico 1.

Seja $Q(Z, t)$ a densidade de fluxo de calor na profundidade $Z$ e no tempo $t$, seja também $Q(Z+d Z, t)$ a densidade de fluxo na profundidade $Z+d Z$, no mesmo instante $t$. $O$ baianço de energia na camada considerada será:

$$
d q=Q(Z, t)-Q(Z+d Z, t)=\frac{-\partial Q}{\partial Z} d Z
$$

O sinal negativo em (1) indica que cada elemento de volume de área unitária e espessura $\mathrm{dZ}$ contido na camada considerada, absorve na unidade tempo uma quantidade $\mathrm{dq}$ de calor, quantidade esta que é responsáve! por todos os processos físicos, químicos e biológicos que aí ocorrem, sendo que o mais signi- ficativo neste caso é o seu aquecimentc. A variação de temperatura de um elemento de volume segundo a calorimetria será:

$$
d q=p C p \frac{\partial T}{\partial t} d z
$$

onde $p$ é densidade média do solo e C seu calor específico.

A quantidade de calor que flui por condução em sólidos homogêneos e isotrópicos pode ser expressa por:

$$
Q=-K(\partial T / \partial Z)
$$

sendo $\mathrm{K}$ a condutividade térmica e $\partial \mathrm{T} / \partial \mathrm{Z}$ o gradiente de temperatura.

Substituindo (3) em (1) e assumindo $\mathrm{K}$ médio, tem-se:

$$
d q=K\left(\frac{\partial^{2} T}{\partial Z^{2}}\right) d z
$$

Igualando (4) com (2) obtém-se:

$$
\frac{\partial T}{\partial t}=D \frac{\partial^{2} T}{\partial Z^{2}}
$$

onde fica definido $D=K / p C$ como a difusividade térmica $\left(\mathrm{cm}^{2} \mathrm{seg}^{-1}\right)$.

A solução clássica da equação diferencial (5) que satisfaz as condições naturais de contorno

$T(0, t)=\bar{T}+T_{0}$ senwt; e $T(\infty, t)=\bar{T}$, é

$T(Z, t)=\bar{T}+T_{0}$ e $-Z \sqrt{\frac{\omega}{2 D}} \operatorname{sen}\left(\omega t-Z \sqrt{\frac{\omega}{2 D}}\right)$

sendo $T$ a temperatura do solo na profundidade $Z$ e tempo $t$; $\bar{T}$ a temperatura média do perfil do solo; $T_{0}$ a amplitude (definida como sendo a metade da diferença entre as temperaturas máxima e mínima em $Z=0$ ), e $\omega$ a velocidade angular da Terra, igual a $\omega=2 \pi / P$, onde $\mathrm{P}$ é o período diurno (24 horas).

A densidade de fluxo de caior ( $Q$ ) equação (3) obtém-se imediatamente derivando parcialmente (6) em função de $Z$, tal como

$$
\begin{aligned}
& Q(Z, t)=-K \frac{\partial}{\partial Z}\left[\bar{T}+T_{0} e\right. \\
& \cdot Z \sqrt{\frac{\omega}{2 D}} \operatorname{sen}\left(\omega t-Z \sqrt{\left.\frac{\omega}{2 D}\right)}\right]
\end{aligned}
$$


que resulta

$$
\begin{aligned}
& Q(Z, t)=K T_{0} \sqrt{\frac{\omega}{D}} e \\
& -Z \sqrt{\frac{\omega}{2 D}} \operatorname{sen}\left(\omega t-Z \sqrt{\frac{\omega}{2 D}}+\pi / 4\right)
\end{aligned}
$$

\section{TEMPERATURAS}

Para a obtenção dos dados de temperatura, seguincio as recomendações da "Organização Meteorológica Mundial", foram instalados 6 (seis) geotermômetros, sendo um deles instalado o mais próximo à superfície e os demais nas profundidades $5,10,20,30$ e $50 \mathrm{~cm}$. As leituras termométricas foram realizadas três vezes ao dia nos horários de 12:00, 18:00 e 24:00 GMT, ou seja, às $8: 00,14: 00$ e 20:00 horas de Manaus. As tabelas IV, V e VI apresentam as temperaturas médias (T) de "décadas" para as seis profundidades, nos três horários do decênio $65-75$. O critério de divisão das "décadas" foi o seguinte: de 1 a 10 , de 11 a 20 e de 21 ao último dia do mês. $O$ intervalo de confiança (IT) que acompanha cada média foi calculado admitindo para teste t o nível de probabilidade de $95 \%$, ou seja, $I C=T+t . s(\bar{T})$, onde $s(\bar{T})$ é o erro da média.

A Tabela VII apresenta as médias mensais para os três horários e para as seis profundidades do decênio 65-75 obtidos de maneira análoga às médias das décadas.

O gráfico II é um exemplo da variação diurna da temperatura (média do mês de setembro) para as seis profundidades obtidas com a equação (6) e o gráfico III representa o comportamento anual da temperatura obtido com médias mensais.

\section{ESTIMATIVA DA AMPLITUDE MENSAL $T_{0}$ EM $\mathrm{Z}=0$}

A estimativa de $T_{0}$ em $Z=0$ foi obtida através da equação das amplitudes, que é pro. veniente da expressão (6) da seguinte forma:

$$
\left.\left.(T)^{\circ}\right)_{Z \neq 0}=(T)\right)_{Z=0} e^{-Z \sqrt{\frac{\omega}{2 D}}} \text { (equa- }
$$

ção das amplitudes) e portanto

$$
\text { (T ) } Z=0=(T)_{0} Z \neq 0 \text { e } Z \sqrt{\frac{\omega}{2 D}}
$$

Analisando os gráficos do actinógrafo do ano de 1976, verificou-se que a densidade máxima de energia radiante tem lugar entre 12:00 e 12:30 horas, admitindo com base na Tabela VII que a temperatura média mensal do perfil é a média das leituras dos três horários na profundidade de $50 \mathrm{~cm}$, e sendo que a difusividade térmica $\left(^{*}\right)$ para esse solo é de $5,0 \times 10^{-3} \mathrm{~cm}^{2} \mathrm{seg}^{-1}$ e a velocidade de propagação da onda de temperatura de $3,0 \mathrm{~cm} \mathrm{~h}^{-1}$ pode-se com razoável aproximação tomar as leituras das 14:00 horas como sendo as das temperaturas máximas a $5 \mathrm{~cm}$ de profunáidade. Os valores estimados da amplitude, assim como os das temperaturas máximas mensais em $\mathrm{Z}=\mathrm{O}$ e das temperaturas médias do perfil encontram-se na Tabela I.

estimativa DA CAMAdA DE "EXTinçÃo" PAFA AS ONDAS DIÁRIAS DE TEMPERATURA

A estimativa da profundidade máxima para a qual a amplitude diária não exceda a $0,1^{\circ} \mathrm{C}\left(\mathrm{T}_{\max }-\overline{\mathrm{T}}=0,1^{\circ} \mathrm{C}\right)$ a temperatura média, definida neste trabalho como camada de extinção, foi obtida com a equação (6) e com os dados estimados de $T_{0}$. Assim resolvendo a equação (6) em termos da profundidade $Z$ para um ponto de máximo, tem-se:

$$
Z=\sqrt{\frac{2 D}{\omega}} \ln \frac{T_{0}}{T \text { max }}-\bar{T}
$$

Os valores calculados de $Z$ encontram-se na Tabela II.

\section{ESTIMATIVA DA QUANTIDADE DE CALOR} ABSORVIDO PELO SOLO

A natureza periódica da variação da densidade de fluxo de calor, equação (7), evidencia períodos alternados de absorção e libera-

[•]-A difusividade e condutividade térmica, assim como a velocidade de propagaçăo da onda de temperatura, foram determinadas em laboratório por técnicas já estabele cidas por Decico (1974). 
ção de calor pelo solo. A quantidade de calor absorvido ou liberado pelo solo num intervalor de tempo t pode ser estimada integrando a equação (7) em $Z=0$, tal como:

$$
\begin{aligned}
& F=\int_{t_{0}}^{t} Q(0, t) d t=T_{0} K \sqrt{\omega / D} \\
& t \\
& \int_{t_{0}}^{t} \operatorname{sen}(\omega t+\pi / 4) d t
\end{aligned}
$$

que resulta

$$
\begin{aligned}
& F=T_{0} K \sqrt{\frac{1}{\omega D}} \\
& {\left[\operatorname{sen}(\omega t-\pi / 4)-\operatorname{sen}\left(\omega t_{0}-\pi / 4\right)\right]}
\end{aligned}
$$

Fazendo $t_{0}=0$. no instante em que o solo começa a ganhar calor $t$ torna-se o tempo de ganho de calor, que devido à latitude de Manaus esse tempo é bem próximo de $P / 2$ $(P=$ período). $E$ assim (11) torna-se, ou seja, a quantidade de calor que flui para o interior do solo do nascer ao pôr do sol é

$$
F=2 T_{0} K \sqrt{\frac{1}{\omega D}} \operatorname{sen} \pi / 4
$$

ou

$$
F=T_{0} K \sqrt{\frac{2}{\omega D}} \mathrm{cal} / \mathrm{cm}^{2}
$$

Para essa estimativa $\mathrm{K}$ assumiu o valor de $2,5 \times 10^{-3} \mathrm{cal} \mathrm{cm}^{-1} \mathrm{seg}^{-1}$. Os dados calculados encontram-se na Tabela III.

\section{RESUltados E DISCUSSÕES}

\section{TEMPERATURAS}

Como já foi observado antes, os dados experimentais de temperatura não foram obtidos de forma contínua como seria de se desejar. Para estimar os valores mais prováveis da temperatura máxima, amplitude e temperatura média do perfil, foram feitas algumas hipóteses. Analisando a Tabela I verif́ica-se que setembro, outubro e novembro são os meses onde o solo atinge as maiores temperaturas: para os demais meses as temperaturas são idênticas.

Analisando as Tabelas IV, V, VI e VII observa-se que às 8:00 horas as temperaturas do perfil do solo são pratícamente iguais para todos os meses, surgindo algumas discrepâncias nas temperaturas às 14:00 e 20:00 horas para os meses de setembro, outubro e novembro.

Devido à latitude de Manaus ( $3^{\circ} 08^{\prime}$ ) seria de se esperar que também para janeiro, fevereiro e março as temperaturas fossem mais altas; isso não ocorre devido ao regime de chu-

\begin{tabular}{|c|c|c|c|c|c|c|c|c|c|c|c|c|}
\hline Meses & Jan. & Fev. & Mar. & Abril & Maio & Jun. & Jul. & Ago. & Set. & Out. & Nov. & Dez. \\
\hline $\mathrm{T}$ & $\begin{array}{r}43,00 \\
\pm 0,3\end{array}$ & $\begin{array}{r}43,10 \\
\pm 0,3\end{array}$ & $\begin{array}{l}43,70 \\
\pm 0,5\end{array}$ & $\begin{array}{r}43,90 \\
\pm 0,3\end{array}$ & $\begin{array}{r}43,40 \\
\pm 0,3\end{array}$ & $\begin{array}{r}43,70 \\
\pm 0.3\end{array}$ & $\begin{array}{r}44,00 \\
\pm 0,4\end{array}$ & $\begin{array}{r}43,90 \\
\pm 0,4\end{array}$ & $\begin{array}{r}45,90 \\
\pm 0,4\end{array}$ & $\begin{array}{r}46,30 \\
\pm 0,5\end{array}$ & $\begin{array}{l}44,50 \\
\pm 0,3\end{array}$ & $\begin{array}{r}43,70 \\
\pm 0,4\end{array}$ \\
\hline $\mathrm{T}_{\text {。 }}$ & 15,50 & 15,50 & 16,10 & 16,40 & 16,20 & 16,50 & 16,90 & 16,30 & 17,80 & 17,70 & 16,20 & 15,50 \\
\hline$\overline{\mathrm{T}}$ & $\begin{array}{r}27,70 \\
\pm 0,2\end{array}$ & $\begin{array}{r}27,60 \\
\pm 0,2\end{array}$ & $\begin{array}{l}27,60 \\
\pm 0,2\end{array}$ & $\begin{array}{l}27,50 \\
\pm 0,2\end{array}$ & $\begin{array}{l}27,20 \\
\pm 0,2\end{array}$ & $\begin{array}{r}27,20 \\
\pm 0,2\end{array}$ & $\begin{array}{l}27,10 \\
\pm 0,2\end{array}$ & $\begin{array}{r}27,60 \\
\pm 0,3\end{array}$ & $\begin{array}{r}28,10 \\
\pm 0,2\end{array}$ & $\begin{array}{r}28,60 \\
\pm 0,2\end{array}$ & $\begin{array}{l}28,30 \\
\pm 0,2\end{array}$ & $\begin{array}{r}28,20 \\
\pm 0,2\end{array}$ \\
\hline
\end{tabular}
vas ou nebulosidade na região.

Fazendo uso da equação (6), o gráfico II mostra a variação diária típica da temperatura para as diversas profundidades. Provavelmente a verdadeira variação seria dada por ondas complexas e não puras como aqui representadas.

TABELA $\mathbf{I}$ - Temperatura máxima mensal $\mathbf{T}$ em $\mathbf{Z}=0$, amplitude $\mathbf{T}_{\text {。 }}$ em $\mathbf{Z}=0$, e temperatura média do perfil $\mathbf{T}$, expressa $\mathrm{em}{ }^{\circ} \mathrm{C}$. 


\begin{tabular}{|c|c|c|c|c|c|c|c|c|c|c|c|c|c|}
\hline \multirow{3}{*}{ HORAS } & \multirow[b]{3}{*}{$\begin{array}{c}\mathrm{Z} \\
(\mathrm{cm}) \\
\end{array}$} & \multirow{2}{*}{\multicolumn{6}{|c|}{$\begin{array}{l}\text { D E C E E N I } \\
\text { FEVEREIRO }\end{array}$}} & \multirow{2}{*}{\multicolumn{3}{|c|}{$\begin{array}{r}65-75 \\
\text { MARÇO }\end{array}$}} & \multirow{2}{*}{\multicolumn{3}{|c|}{ ABRIL }} \\
\hline & & & & & & & & & & & & & \\
\hline & & $1-10$ & $11-20$ & $2 I-3 I$ & $1-10$ & $11-20$ & $21-28$ & $1-10$ & $11-20$ & $21-3 I$ & $1-10$ & $11-20$ & $21-30$ \\
\hline \multirow{6}{*}{$8: 00$} & 0 & $\begin{array}{c}23,3 \pm \\
0,4\end{array}$ & $\begin{array}{c}23,4 \pm \\
0,3\end{array}$ & $\begin{array}{c}23,5 \pm \\
0,4\end{array}$ & $\begin{array}{c}23,3 \pm \\
0,5\end{array}$ & $\begin{array}{c}23,4 \pm \\
0,2\end{array}$ & $\begin{array}{c}23,3 \pm \\
0,3 \\
\end{array}$ & $\begin{array}{c}23,2 \pm \\
0,3 \\
\end{array}$ & $\begin{array}{c}23,5 \pm \\
0,3\end{array}$ & $\begin{array}{c}23,4 \pm \\
0,2 \\
\end{array}$ & $\begin{array}{c}23,5 \pm \\
0,4\end{array}$ & $\begin{array}{c}23,5 \pm \\
0,2\end{array}$ & $\begin{array}{c}23,7 \pm \\
0,6\end{array}$ \\
\hline & 5 & $\begin{array}{c}25,3 \pm \\
0,3 \\
\end{array}$ & $\begin{array}{c}25,0 \pm \\
0,3\end{array}$ & $\begin{array}{c}25,1 \pm \\
0,3 \\
\end{array}$ & $\begin{array}{c}24,9 \pm \\
0,6 \\
\end{array}$ & $\begin{array}{c}25,0 \pm \\
0,3\end{array}$ & $\begin{array}{c}24,9 \pm \\
0,6\end{array}$ & $\begin{array}{c}25,1 \pm \\
0,4\end{array}$ & $\begin{array}{c}25,3 \pm \\
0,3\end{array}$ & $\begin{array}{c}25,3^{ \pm} \\
0,4 \\
\end{array}$ & $\begin{array}{c}25,3 \pm \\
0,3 \\
\end{array}$ & $\begin{array}{c}25,4 \pm \\
0,2 \\
\end{array}$ & $\begin{array}{c}25,3 \pm \\
0,3 \\
\end{array}$ \\
\hline & 10 & $\begin{array}{c}25,7 \pm \\
0,3 \\
\end{array}$ & $\begin{array}{c}25,5 \pm \\
0,4 \\
\end{array}$ & $\begin{array}{c}25,4 \pm \\
0,3 \\
\end{array}$ & $\begin{array}{c}25,6 \pm \\
0,6 \\
\end{array}$ & $\begin{array}{c}24,5 \pm \\
0,4\end{array}$ & $\begin{array}{c}25,6 \pm \\
0,7 \\
\end{array}$ & $\begin{array}{c}25,5 \pm \\
0,6\end{array}$ & $\begin{array}{c}25,7 \pm \\
0,4 \\
\end{array}$ & $\begin{array}{c}25,6 \pm \\
0,6 \\
\end{array}$ & $\begin{array}{c}25,6 \pm \\
0,4\end{array}$ & $\begin{array}{c}25,5 \pm \\
0,7 \\
\end{array}$ & $\begin{array}{c}25,6 \pm \\
0,4 \\
\end{array}$ \\
\hline & 20 & $\begin{array}{c}26,7 \pm \\
0,3 \\
\end{array}$ & $\begin{array}{c}26,5 \pm \\
0,3\end{array}$ & $\begin{array}{c}26,6 \pm \\
0,3\end{array}$ & $\begin{array}{c}26,7^{ \pm} \\
0,4 \\
\end{array}$ & $\begin{array}{c}26,5^{ \pm} \\
0,4\end{array}$ & $\begin{array}{c}26,5 \pm \\
0,5 \\
\end{array}$ & $\begin{array}{c}26,5 \pm \\
0,4 \\
\end{array}$ & $\begin{array}{c}26,7^{ \pm} \\
0,4 \\
\end{array}$ & $\begin{array}{c}26,6 \pm \\
0,4 \\
\end{array}$ & $\begin{array}{c}26,5 \pm \\
0,3\end{array}$ & $\begin{array}{c}26,7^{+} \\
0,3 \\
\end{array}$ & $\begin{array}{c}26,5 \pm \\
0,3 \\
\end{array}$ \\
\hline & 30 & $\begin{array}{c}27,3^{+} \\
0,3 \\
\end{array}$ & $\begin{array}{c}27,1 \pm \\
0,3\end{array}$ & $\begin{array}{c}27,2 \pm \\
0,3 \\
\end{array}$ & \begin{tabular}{|c|}
$27,2 \pm$ \\
0,3 \\
\end{tabular} & $\begin{array}{c}27,1 \pm \\
0,4\end{array}$ & $\begin{array}{c}27,2 \pm \\
0,5\end{array}$ & $\begin{array}{c}27,1 \pm \\
0,4 \\
\end{array}$ & $\begin{array}{c}27,3 \pm \\
0,4\end{array}$ & $\begin{array}{c}27,1 \pm \\
0,4 \\
\end{array}$ & $\begin{array}{c}27,1 \pm \\
0,4\end{array}$ & $\begin{array}{c}27,2 \pm \\
0,2 \\
\end{array}$ & $\begin{array}{c}27,0 \pm \\
0,3 \\
\end{array}$ \\
\hline & 50 & $\begin{array}{c}27,8 \pm \\
0,3\end{array}$ & $\begin{array}{c}27,7^{ \pm} \\
0,3\end{array}$ & $\begin{array}{c}27,7^{ \pm} \\
0,3\end{array}$ & $\begin{array}{c}27,6 \pm \\
0,4\end{array}$ & $\left|\begin{array}{c}27,6 \pm \\
0,3\end{array}\right|$ & $\begin{array}{c}27,6 \pm \\
0,4\end{array}$ & $\begin{array}{c}27,5 \pm \\
0,4\end{array}$ & $\begin{array}{c}27,7 \pm \\
0,3\end{array}$ & $\begin{array}{c}27,5 \pm \\
0,4 \\
\end{array}$ & $\begin{array}{c}27,4^{ \pm} \\
0,4\end{array}$ & $\begin{array}{c}27,6 \pm \\
0,3\end{array}$ & $\begin{array}{c}27,4 \pm \\
0,3\end{array}$ \\
\hline \multirow{6}{*}{$14: 00$} & 0 & $\begin{array}{c}31,0 \pm \\
0,9\end{array}$ & $\begin{array}{c}31,5 \pm \\
1,4\end{array}$ & $\begin{array}{c}30,9 \pm \\
1,0\end{array}$ & $\begin{array}{c}31,0 \pm \\
1,6 \\
\end{array}$ & $\begin{array}{c}31,7 \pm \\
1,0 \\
\end{array}$ & $\begin{array}{c}30,6 \pm \\
1,7 \\
\end{array}$ & $\begin{array}{c}31,0 \pm \\
1,1 \\
\end{array}$ & $\begin{array}{c}32,1 \pm \\
1,4\end{array}$ & $\begin{array}{c}31,9 \pm \\
1,6 \\
\end{array}$ & $\begin{array}{c}31,5 \pm \\
0,9 \\
\end{array}$ & $\begin{array}{c}31,2 \pm \\
1,6 \\
\end{array}$ & $\begin{array}{c}31,6 \pm \\
1,0\end{array}$ \\
\hline & 5 & $\begin{array}{c}28,2 \pm \\
0,7 \\
\end{array}$ & $\begin{array}{c}28,1 \pm \\
0,7\end{array}$ & $\begin{array}{c}28,1 \pm \\
0,5\end{array}$ & $\begin{array}{c}28,1 \pm \\
0,5 \\
\end{array}$ & $\begin{array}{c}28,3^{+} \\
0,7\end{array}$ & $\begin{array}{c}28,0 \pm \\
0,8 \\
\end{array}$ & $\begin{array}{c}28,4^{ \pm} \\
0,7 \\
\end{array}$ & $\begin{array}{c}28,9 \pm \\
0,1\end{array}$ & $\begin{array}{c}28,6 \pm \\
0,5 \\
\end{array}$ & $\begin{array}{c}28,6 \pm \\
0,5\end{array}$ & $\begin{array}{c}28,6 \pm \\
0,5 \\
\end{array}$ & $\begin{array}{c}28,9 \pm \\
0,9\end{array}$ \\
\hline & 10 & $\begin{array}{c}27,7 \pm \\
0,5 \\
\end{array}$ & $\begin{array}{c}27,5 \pm \\
0,6\end{array}$ & $\begin{array}{c}27,6 \pm \\
0,4 \\
\end{array}$ & $\begin{array}{c}28,1 \pm \\
1,3 \\
\end{array}$ & $\begin{array}{c}27,7 \pm \\
0,4\end{array}$ & $\begin{array}{c}27,8 \pm \\
0,6 \\
\end{array}$ & $\begin{array}{c}27,5 \pm \\
0,6\end{array}$ & $\begin{array}{c}27,9 \pm \\
0,5\end{array}$ & $\begin{array}{c}27,9 \pm \\
0,5 \\
\end{array}$ & $\begin{array}{c}28,2 \pm \\
0,8 \\
\end{array}$ & $\begin{array}{c}28,0 \pm \\
0,5\end{array}$ & $\begin{array}{c}27,8 \pm \\
0,4 \\
\end{array}$ \\
\hline & 20 & $\begin{array}{c}27,5 \pm \\
0,3 \\
\end{array}$ & $\begin{array}{c}27,3 \pm \\
0,3 \\
\end{array}$ & $\begin{array}{c}26,6 \pm \\
0,3 \\
\end{array}$ & $\begin{array}{c}27,4 \pm \\
0,5 \\
\end{array}$ & $\begin{array}{c}27,4 \pm \\
0,2\end{array}$ & $\begin{array}{c}27,4 \pm \\
0,4 \\
\end{array}$ & $\begin{array}{c}27,3^{ \pm} \\
0,4\end{array}$ & $\begin{array}{c}27,6 \pm \\
0,3 \\
\end{array}$ & $\begin{array}{c}27,4 \pm \\
0,4 \\
\end{array}$ & $\begin{array}{c}27,4 \pm \\
0,3 \\
\end{array}$ & $\begin{array}{c}27,5 \pm \\
0,2 \\
\end{array}$ & $\begin{array}{c}27,3 \pm \\
0,3 \\
\end{array}$ \\
\hline & 30 & $\begin{array}{c}27,5 \pm \\
0,3 \\
\end{array}$ & $\begin{array}{c}27,3^{ \pm} \\
0,3 \\
\end{array}$ & $\begin{array}{c}27,3 \pm \\
0,3 \\
\end{array}$ & $\begin{array}{c}27,3 \pm \\
0,4 \\
\end{array}$ & $\begin{array}{c}27,3 \pm \\
0,3\end{array}$ & $\begin{array}{c}27,3 \pm \\
0,4 \\
\end{array}$ & $\begin{array}{c}27,3 \pm \\
0,4 \\
\end{array}$ & $\begin{array}{c}27,5 \pm \\
0,3 \\
\end{array}$ & $\begin{array}{c}27,3^{ \pm} \\
0,4 \\
\end{array}$ & $\begin{array}{c}27,3 \pm \\
0,4 \\
\end{array}$ & $\begin{array}{c}27,4 \pm \\
0,2 \\
\end{array}$ & $\begin{array}{c}27,2 \pm \\
0,3 \\
\end{array}$ \\
\hline & 50 & $\begin{array}{c}27,9 \pm \\
0,3 \\
\end{array}$ & $\begin{array}{c}27,8 \pm \\
0,3 \\
\end{array}$ & $\begin{array}{c}27,7 \pm \\
0,3 \\
\end{array}$ & $\begin{array}{c}27,7 \pm \\
0,4 \\
\end{array}$ & $\begin{array}{c}27,7 \pm \\
0,3\end{array}$ & $\begin{array}{c}27,7 \pm \\
0,4 \\
\end{array}$ & $\begin{array}{c}27,7 \pm \\
0,4 \\
\end{array}$ & $\begin{array}{c}27,7 \pm \\
0,3 \\
\end{array}$ & $\begin{array}{c}27,7 \pm \\
0,4 \\
\end{array}$ & $\begin{array}{c}27,6 \pm \\
0,4 \\
\end{array}$ & $\begin{array}{c}27,7 \pm \\
0,3 \\
\end{array}$ & $\begin{array}{c}27,5 \pm \\
0,3 \\
\end{array}$ \\
\hline \multirow{6}{*}{$20: 00$} & 0 & $\begin{array}{c}25,8 \pm \\
0,4 \\
\end{array}$ & $\begin{array}{c}25,7^{ \pm} \\
0,7 \\
\end{array}$ & $\begin{array}{c}25,5 \pm \\
0,5\end{array}$ & $\begin{array}{c}25,7^{ \pm} \\
0,9 \\
\end{array}$ & $\begin{array}{c}25,5 \pm \\
0,5\end{array}$ & $\begin{array}{c}25,8 \pm \\
0,4 \\
\end{array}$ & $\begin{array}{c}25,9 \pm \\
0,8 \\
\end{array}$ & $\begin{array}{c}26,1 \pm \\
1,0 \\
\end{array}$ & $\begin{array}{c}26,1 \pm \\
0,7 \\
\end{array}$ & $\begin{array}{c}26,0 \pm \\
0,7 \\
\end{array}$ & $\begin{array}{c}26,5^{ \pm} \\
0,6 \\
\end{array}$ & $\begin{array}{c}26,6 \pm \\
0,9 \\
\end{array}$ \\
\hline & 5 & $\begin{array}{c}26,5^{ \pm} \\
0,3 \\
\end{array}$ & $\begin{array}{c}26,3 \pm \\
0,3 \\
\end{array}$ & $\begin{array}{c}26,5 \pm \\
0,3\end{array}$ & $\begin{array}{c}26,4^{ \pm} \\
0,6\end{array}$ & {$\left[\begin{array}{c}26,5 \pm \\
0,3\end{array}\right.$} & $\begin{array}{c}26,1 \pm \\
0,6\end{array}$ & $\begin{array}{c}26,3^{ \pm} \\
0,4\end{array}$ & $\mid \begin{array}{c}26,6 \pm \\
0,4\end{array}$ & $\begin{array}{c}26,6 \pm \\
0,5 \\
\end{array}$ & $\begin{array}{c}26,4 \pm \\
0,4\end{array}$ & $\begin{array}{c}26,4 \pm \\
0,7\end{array}$ & $\begin{array}{c}26,4 \pm \\
0,4\end{array}$ \\
\hline & 10 & \begin{tabular}{|c|}
$27,2 \pm$ \\
0,4 \\
\end{tabular} & $\begin{array}{c}26,8 \pm \\
0,5\end{array}$ & \begin{tabular}{|c|}
$27,0 \pm$ \\
0,5 \\
\end{tabular} & \begin{tabular}{|c|}
$27,0 \pm$ \\
0,7 \\
\end{tabular} & $\begin{array}{c}27,0 \pm \\
0,3 \\
\end{array}$ & \begin{tabular}{|c|}
$27,0 \pm$ \\
0,7 \\
\end{tabular} & $\begin{array}{c}27,0 \pm \\
0,5 \\
\end{array}$ & $\begin{array}{c}27,2 \pm \\
0,4 \\
\end{array}$ & $\begin{array}{c}27,1 \pm \\
0,6 \\
\end{array}$ & $\begin{array}{c}27,0 \pm \\
0,4 \\
\end{array}$ & $\begin{array}{c}27,0 \pm \\
0,3 \\
\end{array}$ & $\begin{array}{c}27,0 \pm \\
0,4 \\
\end{array}$ \\
\hline & 20 & \begin{tabular}{|c|}
$27,1 \pm$ \\
0,3 \\
\end{tabular} & $\begin{array}{c}26,9 \pm \\
0,4 \\
\end{array}$ & $\begin{array}{c}27,0 \pm \\
0,3 \\
\end{array}$ & \begin{tabular}{|c|}
$27,0 \pm$ \\
0,4 \\
\end{tabular} & $\begin{array}{c}27,0 \pm \\
0,3 \\
\end{array}$ & $\begin{array}{c}27,0 \pm \\
0,5 \\
\end{array}$ & \begin{tabular}{|c|}
$27,0 \pm$ \\
0,4 \\
\end{tabular} & $\begin{array}{c}27,2 \pm \\
0,3 \\
\end{array}$ & $\begin{array}{c}27,1 \pm \\
0,4\end{array}$ & $\begin{array}{c}27,0 \pm \\
0,3\end{array}$ & $\begin{array}{c}27,2 \pm \\
0,2 \\
\end{array}$ & $\begin{array}{c}26,9 \pm \\
0,3\end{array}$ \\
\hline & 30 & $\begin{array}{c}27,3 \pm \\
0,3 \\
\end{array}$ & $\begin{array}{c}27,1 \pm \\
0,3\end{array}$ & $\begin{array}{c}27,2 \pm \\
0,3 \\
\end{array}$ & $\begin{array}{c}27,2 \pm \\
0,4 \\
\end{array}$ & $\begin{array}{c}27,1 \pm \\
0,3 \\
\end{array}$ & $\begin{array}{c}27,1 \pm \\
0,5 \\
\end{array}$ & $\begin{array}{c}27,1 \pm \\
0,4 \\
\end{array}$ & $\begin{array}{c}27,3 \pm \\
0,3 \\
\end{array}$ & $\begin{array}{c}27,0 \pm \\
0,4 \\
\end{array}$ & $\begin{array}{c}27,1 \pm \\
0,3\end{array}$ & $\begin{array}{c}27,2 \pm \\
0,2 \\
\end{array}$ & $\begin{array}{c}27,0 \pm \\
0,3 \\
\end{array}$ \\
\hline & 50 & $\begin{array}{c}27,8 \pm \\
0,3\end{array}$ & $\begin{array}{c}27,6 \pm \\
0,3\end{array}$ & $\begin{array}{c}27,6 \pm \\
0,3\end{array}$ & $\begin{array}{c}27,6 \pm \\
0,4\end{array}$ & $\begin{array}{c}27,5 \pm \\
0,3\end{array}$ & $\begin{array}{c}27,6 \pm \\
0,4\end{array}$ & $\begin{array}{c}27,7^{ \pm} \\
0,5\end{array}$ & $\begin{array}{c}27,7^{ \pm} \\
0,3\end{array}$ & $\begin{array}{c}27,5 \pm \\
0,4\end{array}$ & $\begin{array}{c}27,4 \pm \\
0,4\end{array}$ & $\begin{array}{c}27,6 \pm \\
0,3\end{array}$ & $\begin{array}{c}27,4 \pm \\
0,3\end{array}$ \\
\hline
\end{tabular}

TABELA IV - Temperatura média de décadas, decênio 65-75, nas profundidades de 0,5 , $10,20,30$ e $50 \mathrm{~cm}$. 


\begin{tabular}{|c|c|c|c|c|c|c|c|c|c|c|c|c|c|}
\hline \multirow{3}{*}{ HORAS } & \multirow[b]{3}{*}{$\begin{array}{c}\mathrm{Z} \\
(\mathrm{cm}) \\
\end{array}$} & \multicolumn{12}{|c|}{ DEC EN I O $65-75$} \\
\hline & & \multicolumn{3}{|c|}{ MAIO } & \multicolumn{3}{|c|}{ JUNHO } & \multicolumn{3}{|c|}{\begin{tabular}{|r|} 
JULHO \\
\end{tabular}} & \multicolumn{3}{|c|}{ AGOSTO } \\
\hline & & $1-10$ & $11-20$ & $21-31$ & $1-10$ & $11-20$ & $21-30$ & $1-10$ & $11-20$ & $21-31$ & $1-10$ & $11-20$ & $21-31$ \\
\hline \multirow{6}{*}{$8: 00$} & 0 & $\begin{array}{c}23,2 \pm \\
0,3\end{array}$ & $\begin{array}{c}23,2 \pm \\
0,4 \\
\end{array}$ & $\begin{array}{c}23,4 \pm \\
0,3 \\
\end{array}$ & $\begin{array}{c}23,2 \pm \\
0,3\end{array}$ & $\begin{array}{c}23,1 \pm \\
0,3\end{array}$ & $\begin{array}{c}22,9 \pm \\
0,3 \\
\end{array}$ & $\begin{array}{c}22,7 \pm \\
0,3 \\
\end{array}$ & $\begin{array}{c}22,5 \pm \\
0,2\end{array}$ & $\begin{array}{c}23,0 \pm \\
0,4\end{array}$ & $\begin{array}{c}22,8 \pm \\
0,4 \\
\end{array}$ & $\begin{array}{c}22,9 \pm \\
0,3 \\
\end{array}$ & $\begin{array}{c}23,3 \pm \\
0,3\end{array}$ \\
\hline & 5 & $\begin{array}{c}25,1 \pm \\
0,3 \\
\end{array}$ & $\begin{array}{c}25,1 \pm \\
0,4\end{array}$ & $\begin{array}{c}25,3 \pm \\
0,4 \\
\end{array}$ & $\begin{array}{c}25,1 \pm \\
0,4\end{array}$ & $\begin{array}{c}25,2 \pm \\
0,3\end{array}$ & $\begin{array}{c}25,0 \pm \\
0,4 \\
\end{array}$ & $\begin{array}{c}24,6 \pm \\
0,3 \\
\end{array}$ & $\begin{array}{c}24,7 \pm \\
0,1 \\
\end{array}$ & $\begin{array}{c}25,0 \pm \\
0,2\end{array}$ & $\begin{array}{c}25,0 \pm \\
0,3 \\
\end{array}$ & $\begin{array}{c}24,9 \pm \\
0,4 \\
\end{array}$ & $\begin{array}{c}25,4 \pm \\
0,3 \\
\end{array}$ \\
\hline & 10 & $\begin{array}{c}25,1 \pm \\
0,5 \\
\end{array}$ & $\begin{array}{c}25,4 \pm \\
0,4 \\
\end{array}$ & $\begin{array}{c}25,5 \pm \\
0,5 \\
\end{array}$ & $\begin{array}{c}25,3^{+} \\
0,5 \\
\end{array}$ & $\begin{array}{c}25,4^{+} \\
0,4 \\
\end{array}$ & $\begin{array}{c}25,3^{ \pm} \\
0,4 \\
\end{array}$ & $\begin{array}{c}25,2 \pm \\
0,3 \\
\end{array}$ & $\begin{array}{c}25,0 \pm \\
0,2 \\
\end{array}$ & $\begin{array}{c}25,3^{ \pm} \\
0,3 \\
\end{array}$ & $\begin{array}{c}25,4 \pm \\
0,3 \\
\end{array}$ & $\begin{array}{c}25,6^{ \pm} \\
0,4 \\
\end{array}$ & $\begin{array}{c}25,7 \pm \\
0,7 \\
\end{array}$ \\
\hline & 20 & $\begin{array}{c}26,2 \pm \\
0,4 \\
\end{array}$ & $\begin{array}{c}26,4^{ \pm} \\
0,3 \\
\end{array}$ & $\begin{array}{c}26,4^{ \pm} \\
0,4 \\
\end{array}$ & $\begin{array}{c}26,2 \pm \\
0,5 \\
\end{array}$ & $\begin{array}{c}26,3^{ \pm} \\
0,3 \\
\end{array}$ & $\begin{array}{c}26,0 \pm \\
0,6 \\
\end{array}$ & $\begin{array}{c}26,2 \pm \\
0,3 \\
\end{array}$ & $\begin{array}{c}26,0 \pm \\
0,1 \\
\end{array}$ & $\begin{array}{c}26,4 \pm \\
0,1 \\
\end{array}$ & $\begin{array}{c}26,5 \pm \\
0,2 \\
\end{array}$ & $\begin{array}{c}26,6 \pm \\
0,3 \\
\end{array}$ & $\begin{array}{c}26,7 \pm \\
0,7 \\
\end{array}$ \\
\hline & 30 & $\begin{array}{c}26,7 \pm \\
0,7 \\
\end{array}$ & $\begin{array}{c}26,9 \pm \\
0,3 \\
\end{array}$ & $\begin{array}{c}26,9 \pm \\
0,4 \\
\end{array}$ & $\begin{array}{c}26,8 \pm \\
0,5\end{array}$ & $\begin{array}{c}26,9 \pm \\
0,3 \\
\end{array}$ & $\begin{array}{c}26,8 \pm \\
0,3 \\
\end{array}$ & $\begin{array}{c}26,7 \pm \\
0,2 \\
\end{array}$ & $\begin{array}{c}26,5 \pm \\
0,1\end{array}$ & $\begin{array}{c}26,9 \pm \\
0,1\end{array}$ & $\begin{array}{c}27,1 \pm \\
0,2\end{array}$ & $\begin{array}{c}27,0 \pm \\
0,8\end{array}$ & $\begin{array}{c}27,6 \pm \\
0,2\end{array}$ \\
\hline & 50 & $\begin{array}{c}27,1 \pm \\
0,4 \\
\end{array}$ & $\begin{array}{c}27,3 \pm \\
0,2 \\
\end{array}$ & $\begin{array}{c}27,3 \pm \\
0,3 \\
\end{array}$ & $\begin{array}{c}27,2 \pm \\
0,4 \\
\end{array}$ & $\begin{array}{c}27,3^{ \pm} \\
0,3 \\
\end{array}$ & $\begin{array}{c}27,2 \pm \\
0,3 \\
\end{array}$ & $\begin{array}{c}27,5 \pm \\
0,2 \\
\end{array}$ & $\begin{array}{c}27,0 \pm \\
0,2 \\
\end{array}$ & $\begin{array}{c}27,3^{ \pm} \\
0,2 \\
\end{array}$ & $\begin{array}{c}27,5 \pm \\
0,2 \\
\end{array}$ & $\begin{array}{c}27,6 \pm \\
0,3 \\
\end{array}$ & $\begin{array}{c}27,9 \pm \\
0,2 \\
\end{array}$ \\
\hline \multirow{6}{*}{$14: 00$} & 0 & $\begin{array}{c}30,7 \pm \\
0,8 \\
\end{array}$ & $\begin{array}{c}32,3^{ \pm} \\
1,4\end{array}$ & $\begin{array}{c}32,5 \pm \\
1,3 \\
\end{array}$ & $\begin{array}{c}32,6 \pm \\
1,4 \\
\end{array}$ & $\begin{array}{c}32,3 \pm \\
2,4 \\
\end{array}$ & $\begin{array}{c}32,9 \pm \\
1,2 \\
\end{array}$ & $\begin{array}{c}32,3^{ \pm} \\
1,2 \\
\end{array}$ & $\begin{array}{c}32,5 \pm \\
1,4 \\
\end{array}$ & $\begin{array}{c}35,5 \pm \\
1,0\end{array}$ & $\begin{array}{c}34,7 \pm \\
0,9 \\
\end{array}$ & $\begin{array}{c}35,9 \pm \\
1,5 \\
\end{array}$ & $\begin{array}{c}35,8 \pm \\
1,7 \\
\end{array}$ \\
\hline & 5 & $\begin{array}{c}28,1 \pm \\
0,6 \\
\end{array}$ & $\begin{array}{c}28,4^{ \pm} \\
0,6 \\
\end{array}$ & $\begin{array}{c}28,7 \pm \\
0,8 \\
\end{array}$ & $\begin{array}{c}28,5 \pm \\
0,6\end{array}$ & $\begin{array}{c}28,7^{ \pm} \\
0,5 \\
\end{array}$ & $\begin{array}{c}28,7^{ \pm} \\
0,7 \\
\end{array}$ & $\begin{array}{c}28,6^{ \pm} \\
0,7 \\
\end{array}$ & $\begin{array}{c}28,2 \pm \\
0,7 \\
\end{array}$ & $\begin{array}{c}29,5 \pm \\
0,9 \\
\end{array}$ & $\begin{array}{c}29,3^{ \pm} \\
0,9 \\
\end{array}$ & $\begin{array}{c}29,7^{ \pm} \\
1,0 \\
\end{array}$ & $\begin{array}{c}30,0 \pm \\
0,8\end{array}$ \\
\hline & 10 & $\begin{array}{c}27,5 \pm \\
0,5 \\
\end{array}$ & $\begin{array}{c}27,8 \pm \\
0,5 \\
\end{array}$ & $\begin{array}{c}27,8 \pm \\
0,6 \\
\end{array}$ & $\begin{array}{c}27,7^{ \pm} \\
0,5 \\
\end{array}$ & $\begin{array}{c}27,9 \pm \\
0,5 \\
\end{array}$ & $\begin{array}{c}27,7 \pm \\
0,4 \\
\end{array}$ & $\begin{array}{c}27,5 \pm \\
0,5 \\
\end{array}$ & $\begin{array}{c}27,5 \pm \\
0,4 \\
\end{array}$ & $\begin{array}{c}28,2 \pm \\
0,5\end{array}$ & $\begin{array}{c}28,4 \pm \\
0,6 \\
\end{array}$ & $\begin{array}{c}28,5 \pm \\
0,6\end{array}$ & $\begin{array}{c}28,8 \pm \\
0,4 \\
\end{array}$ \\
\hline & 20 & $\begin{array}{c}27,3^{ \pm} \\
0,6 \\
\end{array}$ & $\begin{array}{c}27,2 \pm \\
0,3 \\
\end{array}$ & $\begin{array}{l}27,2 \pm \\
.0,4 \\
\end{array}$ & $\begin{array}{c}27,1 \pm \\
0,4\end{array}$ & $\begin{array}{c}27,2 \pm \\
0,2 \\
\end{array}$ & $\begin{array}{c}27,1 \pm \\
0,2 \\
\end{array}$ & $\begin{array}{c}26,9 \pm \\
0,2 \\
\end{array}$ & $\begin{array}{c}26,8 \pm \\
0,3 \\
\end{array}$ & $\begin{array}{c}27,3 \pm \\
0,2 \\
\end{array}$ & $\begin{array}{c}27,5 \pm \\
0,3 \\
\end{array}$ & $\begin{array}{c}27,7 \pm \\
0,3 \\
\end{array}$ & $\begin{array}{c}28,1 \pm \\
0,2\end{array}$ \\
\hline & 30 & $\begin{array}{c}26,9 \pm \\
0,3 \\
\end{array}$ & $\begin{array}{c}27,0 \pm \\
0,2 \\
\end{array}$ & $\begin{array}{c}27,1 \pm \\
0,3 \\
\end{array}$ & $\begin{array}{c}26,9 \pm \\
0,4 \\
\end{array}$ & $\begin{array}{c}27,1 \pm \\
0,3 \\
\end{array}$ & $\begin{array}{c}27,0 \pm \\
0,2 \\
\end{array}$ & $\begin{array}{c}26,8 \pm \\
0,2 \\
\end{array}$ & $\begin{array}{c}26,7 \pm \\
0,2 \\
\end{array}$ & $\begin{array}{c}27,1 \pm \\
0,1 \\
\end{array}$ & $\begin{array}{c}27,2 \pm \\
0,2 \\
\end{array}$ & $\begin{array}{c}27,5 \pm \\
0,3 \\
\end{array}$ & $\begin{array}{c}27,8 \pm \\
0,2 \\
\end{array}$ \\
\hline & 50 & $\begin{array}{c}27,3^{ \pm} \\
0,4 \\
\end{array}$ & $\begin{array}{c}27,4 \pm \\
0,2 \\
\end{array}$ & $\begin{array}{c}27,4 \pm \\
0,3 \\
\end{array}$ & $\begin{array}{c}27,3 \pm \\
0,4 \\
\end{array}$ & $\begin{array}{c}27,3 \pm \\
0,3 \\
\end{array}$ & $\begin{array}{c}27,4 \pm \\
0,3 \\
\end{array}$ & $\begin{array}{c}27,3 \pm \\
0,2 \\
\end{array}$ & $\begin{array}{c}27,1 \pm \\
0,2\end{array}$ & $\begin{array}{c}27,4 \pm \\
0,2 \\
\end{array}$ & $\begin{array}{c}27,6 \pm \\
0,2 \\
\end{array}$ & $\begin{array}{c}27,7^{ \pm} \\
0,3 \\
\end{array}$ & $\begin{array}{c}28,0 \pm \\
0,2\end{array}$ \\
\hline \multirow{6}{*}{$20: 00$} & 0 & $\begin{array}{c}26,2^{ \pm} \\
0,4 \\
\end{array}$ & $\begin{array}{c}26,9 \pm \\
0,8 \\
\end{array}$ & $\begin{array}{c}27,7 \pm \\
1,0 \\
\end{array}$ & $\begin{array}{c}26,5 \pm \\
0,9 \\
\end{array}$ & $\begin{array}{c}27,6 \pm \\
0,9 \\
\end{array}$ & $\begin{array}{c}28,6 \pm \\
1,3 \\
\end{array}$ & $\begin{array}{c}27,3 \pm \\
1,3 \\
\end{array}$ & $\begin{array}{c}27,3 \pm \\
0,9 \\
\end{array}$ & $\begin{array}{c}28,7^{ \pm} \\
0,7 \\
\end{array}$ & $\begin{array}{c}29,3 \pm \\
1,3 \\
\end{array}$ & $\begin{array}{c}29,3 \pm \\
1,3 \\
\end{array}$ & $\begin{array}{c}30,0 \pm \\
1,2\end{array}$ \\
\hline & 5 & $\begin{array}{c}26,2 \pm \\
0,4 \\
\end{array}$ & $\begin{array}{c}26,4 \pm \\
0,4 \\
\end{array}$ & $\begin{array}{c}26,4^{ \pm} \\
0,6 \\
\end{array}$ & $\begin{array}{c}26,2 \pm \\
0,5 \\
\end{array}$ & $\begin{array}{c}26,4^{ \pm} \\
0,3 \\
\end{array}$ & $\begin{array}{c}26,3^{ \pm} \\
0,2 \\
\end{array}$ & $\begin{array}{c}26,1 \pm \\
0,2 \\
\end{array}$ & $\begin{array}{c}26,0 \pm \\
0,3 \\
\end{array}$ & $\begin{array}{c}26,7^{+} \\
0,3 \\
\end{array}$ & $\begin{array}{c}26,6^{ \pm} \\
0,6 \\
\end{array}$ & $\begin{array}{c}26,8 \pm \\
0,4 \\
\end{array}$ & $\begin{array}{c}27,0 \pm \\
0,3 \\
\end{array}$ \\
\hline & 10 & $\begin{array}{c}26,7 \pm \\
0,4 \\
\end{array}$ & $\begin{array}{c}26,8 \pm \\
0,4 \\
\end{array}$ & $\begin{array}{c}26,9 \pm \\
0,6 \\
\end{array}$ & $\begin{array}{c}27,7 \pm \\
0,5 \\
\end{array}$ & $\begin{array}{c}27,0 \pm \\
0,3 \\
\end{array}$ & $\begin{array}{c}26,8 \pm \\
0,3 \\
\end{array}$ & $\begin{array}{c}26,7 \pm \\
0,3 \\
\end{array}$ & $\begin{array}{c}26,3^{ \pm} \\
0,6 \\
\end{array}$ & $\begin{array}{c}27,1 \pm \\
0,3 \\
\end{array}$ & $\begin{array}{c}27,3 \pm \\
0,5 \\
\end{array}$ & $\begin{array}{c}27,3 \pm \\
0,5 \\
\end{array}$ & $\begin{array}{c}27,4 \pm \\
0,4 \\
\end{array}$ \\
\hline & 20 & $\begin{array}{c}26,6 \pm \\
0,3 \\
\end{array}$ & $\begin{array}{c}26,8 \pm \\
0,3 \\
\end{array}$ & $\begin{array}{c}26,9 \pm \\
0,4 \\
\end{array}$ & $\begin{array}{c}26,8 \pm \\
0,4 \\
\end{array}$ & $\begin{array}{c}26,9 \pm \\
0,3 \\
\end{array}$ & $\begin{array}{c}26,8 \pm \\
0,2 \\
\end{array}$ & $\begin{array}{c}26,5 \pm \\
0,4 \\
\end{array}$ & $\begin{array}{c}26,5 \pm \\
0,1 \\
\end{array}$ & $\begin{array}{c}27,0 \pm \\
0,2 \\
\end{array}$ & $\begin{array}{c}27,2 \pm \\
0,2 \\
\end{array}$ & $\begin{array}{c}27,3 \pm \\
0,3 \\
\end{array}$ & $\begin{array}{c}27,7 \pm \\
0,2 \\
\end{array}$ \\
\hline & 30 & $\begin{array}{c}26,7 \pm \\
0,3 \\
\end{array}$ & $\begin{array}{c}26,9 \pm \\
0,2 \\
\end{array}$ & $\begin{array}{c}27,0 \pm \\
0,4 \\
\end{array}$ & \begin{tabular}{|c|}
$26,8 \pm$ \\
0,4 \\
\end{tabular} & $\begin{array}{c}26,9 \pm \\
0,3 \\
\end{array}$ & $\begin{array}{c}26,8 \pm \\
0,3 \\
\end{array}$ & $\begin{array}{c}26,7^{ \pm} \\
0,2 \\
\end{array}$ & $\begin{array}{c}26,5 \pm \\
0,2 \\
\end{array}$ & $\begin{array}{c}27,1 \pm \\
0,2 \\
\end{array}$ & $\begin{array}{c}27,1 \pm \\
0,2\end{array}$ & $\begin{array}{c}27,3 \pm \\
0,3\end{array}$ & $\begin{array}{c}27,6 \pm \\
0,1 \\
\end{array}$ \\
\hline & 50 & $\begin{array}{c}27,1 \pm \\
0,3 \\
\end{array}$ & $\begin{array}{c}27,3^{ \pm} \\
0,2 \\
\end{array}$ & $\begin{array}{c}27,4 \pm \\
0,3 \\
\end{array}$ & $\begin{array}{c}27,2 \pm \\
0,4 \\
\end{array}$ & $\begin{array}{c}27,4 \pm \\
0,3 \\
\end{array}$ & $\begin{array}{c}27,2 \pm \\
0,3 \\
\end{array}$ & $\begin{array}{c}27,1 \pm \\
0,2 \\
\end{array}$ & $\begin{array}{c}27,0 \pm \\
0,1\end{array}$ & $\begin{array}{c}27,2 \pm \\
0,2 \\
\end{array}$ & $\begin{array}{c}27,4 \pm \\
0,2 \\
\end{array}$ & $\begin{array}{c}27,6 \pm \\
0,3 \\
\end{array}$ & $\begin{array}{c}27,8 \pm \\
0,2 \\
\end{array}$ \\
\hline
\end{tabular}

TABELA V - Temperatura média de décadas, decênio 65-75, nas profundidades de 0,5, $10,20,30$ e $50 \mathrm{~cm}$. 


\begin{tabular}{|c|c|c|c|c|c|c|c|c|c|c|c|c|c|}
\hline \multirow{3}{*}{ HORAS } & \multirow[b]{3}{*}{$\begin{array}{c}\mathrm{Z} \\
(\mathrm{cm}) \\
\end{array}$} & \multirow{2}{*}{\multicolumn{6}{|c|}{$\begin{array}{l}\text { D E C E N I O } \\
\text { OUTU BRO }\end{array}$}} & \multirow{2}{*}{\multicolumn{3}{|c|}{$\frac{65-75}{\text { NOVEMBRO }}$}} & \multirow{2}{*}{\multicolumn{3}{|c|}{ DEZEMBRO }} \\
\hline & & & & & & & & & & & & & \\
\hline & & $1-10$ & $11-20$ & $21-30$ & $1-10$ & $11-20$ & $21-31$ & $1-10$ & $11-20$ & $21-30$ & $1-10$ & $11-20$ & $21-31$ \\
\hline \multirow{6}{*}{$8: 00$} & 0 & $\begin{array}{c}23,2 \pm \\
0,5 \\
\end{array}$ & $\begin{array}{c}23,3 \pm \\
0,5 \\
\end{array}$ & $\begin{array}{c}23,5 \pm \\
0,4\end{array}$ & $\begin{array}{c}23,9 \pm \\
0,5 \\
\end{array}$ & $\begin{array}{c}23,4 \pm \\
0,3 \\
\end{array}$ & $\begin{array}{c}23,7 \pm \\
0,3 \\
\end{array}$ & $\begin{array}{c}23,9 \pm \\
0,5\end{array}$ & $\begin{array}{c}24,1 \pm \\
0,5\end{array}$ & $\begin{array}{c}23,7 \pm \\
0,4 \\
\end{array}$ & $\begin{array}{c}23,5 \pm \\
0,3 \\
\end{array}$ & $\begin{array}{c}23,8 \pm \\
0,3 \\
\end{array}$ & $\begin{array}{c}23,4 \pm \\
0,4 \\
\end{array}$ \\
\hline & 5 & $\begin{array}{c}25,4 \pm \\
0,4 \\
\end{array}$ & $\begin{array}{c}25,9 \pm \\
0,4 \\
\end{array}$ & $\begin{array}{c}25,5 \pm \\
0,7 \\
\end{array}$ & $\begin{array}{c}26,3 \pm \\
0,4 \\
\end{array}$ & $\begin{array}{c}26,0 \pm \\
0,3 \\
\end{array}$ & $\begin{array}{c}26,0 \pm \\
0,3\end{array}$ & $\begin{array}{c}26,1 \pm \\
0,3\end{array}$ & $\begin{array}{c}26,0 \pm \\
0,3 \\
\end{array}$ & $\begin{array}{c}25,9 \pm \\
0,4 \\
\end{array}$ & $\begin{array}{c}25,8 \pm \\
0,3\end{array}$ & $\begin{array}{c}25,8 \pm \\
0,4\end{array}$ & $\begin{array}{c}25,5 \pm \\
0,3\end{array}$ \\
\hline & 10 & $\begin{array}{c}25,9 \pm \\
0,5 \\
\end{array}$ & $\begin{array}{c}26,1 \pm \\
0,5 \\
\end{array}$ & $\begin{array}{c}26,2 \pm \\
0,4 \\
\end{array}$ & $\begin{array}{c}26,8 \pm \\
0,4\end{array}$ & $\begin{array}{c}26,5 \pm \\
0,3 \\
\end{array}$ & $\begin{array}{c}26,5 \pm \\
0,4 \\
\end{array}$ & $\begin{array}{c}26,4^{ \pm} \\
0,4 \\
\end{array}$ & $\begin{array}{c}26,4 \pm \\
0,3 \\
\end{array}$ & $\begin{array}{c}26,1 \pm \\
0,4 \\
\end{array}$ & $\begin{array}{c}26,1 \pm \\
0,3 \\
\end{array}$ & $\begin{array}{c}26,3 \pm \\
0,5 \\
\end{array}$ & $\begin{array}{c}25,8 \pm \\
0,3 \\
\end{array}$ \\
\hline & 20 & $\begin{array}{c}26,9 \pm \\
0,3 \\
\end{array}$ & $\begin{array}{c}27,2 \pm \\
0,3 \\
\end{array}$ & $\begin{array}{c}27,2 \pm \\
0,3 \\
\end{array}$ & $\begin{array}{c}27,8 \pm \\
0,3 \\
\end{array}$ & $\begin{array}{c}27,5 \pm \\
0,3 \\
\end{array}$ & $\begin{array}{c}27,5 \pm \\
0,4 \\
\end{array}$ & $\begin{array}{c}27,4 \pm \\
0,3 \\
\end{array}$ & $\begin{array}{c}27,3 \pm \\
0,3 \\
\end{array}$ & $\begin{array}{c}27,2 \pm \\
0,4 \\
\end{array}$ & $\begin{array}{c}27,2 \pm \\
0,2 \\
\end{array}$ & $\begin{array}{c}27,2 \pm \\
0,3 \\
\end{array}$ & $\begin{array}{c}26,7 \pm \\
0,5 \\
\end{array}$ \\
\hline & 30 & $\begin{array}{c}27,5 \pm \\
0,1 \\
\end{array}$ & $\begin{array}{c}27,6 \pm \\
0,7 \\
\end{array}$ & $\begin{array}{c}27,9 \pm \\
0,3 \\
\end{array}$ & $\begin{array}{c}28,4 \pm \\
0,4 \\
\end{array}$ & $\begin{array}{c}28,3 \pm \\
0,2 \\
\end{array}$ & $\begin{array}{c}28,2 \pm \\
0,3 \\
\end{array}$ & $\begin{array}{c}28,0 \pm \\
0,3 \\
\end{array}$ & $\begin{array}{c}27,9 \pm \\
0,2 \\
\end{array}$ & $\begin{array}{c}27,8 \pm \\
0,4 \\
\end{array}$ & $\begin{array}{c}27,8 \pm \\
0,4 \\
\end{array}$ & $\begin{array}{c}27,9 \pm \\
0,2\end{array}$ & $\begin{array}{c}27,5 \pm \\
0,2 \\
\end{array}$ \\
\hline & 50 & $\begin{array}{c}27,9 \pm \\
0,2 \\
\end{array}$ & $\begin{array}{c}28,2 \pm \\
0,3 \\
\end{array}$ & $\begin{array}{c}28,2 \pm \\
0,3 \\
\end{array}$ & $\begin{array}{c}28,6 \pm \\
0,4 \\
\end{array}$ & $\begin{array}{c}28,6 \pm \\
0,3 \\
\end{array}$ & $\begin{array}{c}28,6 \pm \\
0,3 \\
\end{array}$ & $\begin{array}{c}28,5 \pm \\
0,2 \\
\end{array}$ & $\begin{array}{c}28,4^{ \pm} \\
0,2 \\
\end{array}$ & $\begin{array}{c}28,1 \pm \\
0,5 \\
\end{array}$ & $\begin{array}{c}28,3^{ \pm} \\
0,3 \\
\end{array}$ & $\begin{array}{c}28,3 \pm \\
0,3 \\
\end{array}$ & $\begin{array}{c}27,7^{ \pm} \\
0,6 \\
\end{array}$ \\
\hline \multirow{6}{*}{$14: 00$} & 0 & $\begin{array}{c}35,6 \pm \\
2,0 \\
\end{array}$ & $\begin{array}{c}36,6 \pm \\
1,5\end{array}$ & $\begin{array}{c}35,9 \pm \\
1,6 \\
\end{array}$ & $\begin{array}{c}36,5 \pm \\
2,0 \\
\end{array}$ & $\begin{array}{c}36,1 \pm \\
1,2 \\
\end{array}$ & $\begin{array}{c}35,5 \pm \\
1,2 \\
\end{array}$ & $\begin{array}{c}33,7^{ \pm} \\
1,0 \\
\end{array}$ & $\begin{array}{c}33,7^{ \pm} \\
1,0 \\
\end{array}$ & $\begin{array}{c}32,4 \pm \\
1,1 \\
\end{array}$ & $\begin{array}{c}33,3 \pm \\
1,6\end{array}$ & $\begin{array}{c}32,5 \pm \\
1,4 \\
\end{array}$ & $\begin{array}{c}31,5 \pm \\
0,7 \\
\end{array}$ \\
\hline & 5 & $\begin{array}{c}29,8 \pm \\
0,7 \\
\end{array}$ & $\begin{array}{c}30,2 \pm \\
0,8 \\
\end{array}$ & $\begin{array}{c}30,0 \pm \\
1,0 \\
\end{array}$ & $\begin{array}{c}30,5 \pm \\
1,1\end{array}$ & $\begin{array}{c}30,4 \pm \\
1,1 \\
\end{array}$ & $\begin{array}{c}30,1 \pm \\
0,9\end{array}$ & $\begin{array}{c}29,2 \pm . \\
0,5\end{array}$ & $\begin{array}{c}29,2 \pm \\
0,8\end{array}$ & $\begin{array}{c}28,7 \pm \\
0,7 \\
\end{array}$ & $\begin{array}{c}29,1 \pm \\
1,1 \\
\end{array}$ & $\begin{array}{c}28,5 \pm \\
0,5\end{array}$ & $\begin{array}{c}28,2 \pm \\
0,4 \\
\end{array}$ \\
\hline & 10 & $\begin{array}{c}28,9 \pm \\
0,5 \\
\end{array}$ & $\begin{array}{c}29,4 \pm \\
1,1 \\
\end{array}$ & $\begin{array}{c}29,1 \pm \\
0,6 \\
\end{array}$ & $\begin{array}{c}29,6 \pm \\
0,8 \\
\end{array}$ & $\begin{array}{c}29,1 \pm \\
0,6 \\
\end{array}$ & $\begin{array}{c}28,8 \pm \\
0,5 \\
\end{array}$ & $\begin{array}{c}28,5 \pm \\
0,3 \\
\end{array}$ & $\begin{array}{c}28,5 \pm \\
0,4 \\
\end{array}$ & $\begin{array}{c}28,1 \pm \\
0,4 \\
\end{array}$ & $\begin{array}{c}28,2 \pm \\
0,5 \\
\end{array}$ & $\begin{array}{c}28,2 \pm \\
0,5 \\
\end{array}$ & $\begin{array}{c}27,8 \pm \\
0,2 \\
\end{array}$ \\
\hline & 20 & $\begin{array}{c}28,0 \pm \\
0,3 \\
\end{array}$ & $\begin{array}{c}28,3 \pm \\
0,3 \\
\end{array}$ & $\begin{array}{c}28,3 \pm \\
0,3 \\
\end{array}$ & $\begin{array}{c}28,9 \pm \\
0,5 \\
\end{array}$ & $\begin{array}{c}28,6 \pm \\
0,4 \\
\end{array}$ & $\begin{array}{c}28,6 \pm \\
0,4 \\
\end{array}$ & $\begin{array}{c}28,2 \pm \\
0,2\end{array}$ & $\begin{array}{c}28,2 \pm \\
0,2 \\
\end{array}$ & $\begin{array}{c}28,0 \pm \\
0,4 \\
\end{array}$ & $\begin{array}{c}28,1 \pm \\
0,3\end{array}$ & $\begin{array}{c}28,0 \pm \\
0,4 \\
\end{array}$ & $\begin{array}{c}27,6 \pm \\
0,2 \\
\end{array}$ \\
\hline & 30 & $\begin{array}{c}27,7^{ \pm} \\
0,2 \\
\end{array}$ & $\begin{array}{c}27,8 \pm \\
0,7 \\
\end{array}$ & $\begin{array}{c}28,1 \pm \\
0,3 \\
\end{array}$ & $\begin{array}{c}28,6 \pm \\
0,4 \\
\end{array}$ & $\begin{array}{c}28,5^{ \pm} \\
0,3 \\
\end{array}$ & $\begin{array}{c}28,5 \pm \\
0,3 \\
\end{array}$ & $\begin{array}{c}28,2 \pm \\
0,2 \\
\end{array}$ & $\begin{array}{c}28,1 \pm \\
0,2 \\
\end{array}$ & $\begin{array}{c}27,8 \pm \\
0,4 \\
\end{array}$ & $\begin{array}{c}27,8 \pm \\
0,2 \\
\end{array}$ & $\begin{array}{c}27,8 \pm \\
0,3 \\
\end{array}$ & $\begin{array}{c}27,6 \pm \\
0,2 \\
\end{array}$ \\
\hline & 50 & $\begin{array}{c}28,0 \pm \\
0,2 \\
\end{array}$ & $\begin{array}{c}28,3 \pm \\
0,3 \\
\end{array}$ & $\begin{array}{c}28,3 \pm \\
0,3 \\
\end{array}$ & $\begin{array}{c}28,7 \pm \\
0,3 \\
\end{array}$ & $\begin{array}{c}28,8 \pm \\
0,3 \\
\end{array}$ & $\begin{array}{c}28,7 \pm \\
0,3 \\
\end{array}$ & $\begin{array}{c}28,6 \pm \\
0,3 \\
\end{array}$ & $\begin{array}{c}28,5 \pm \\
0,2 \\
\end{array}$ & $\begin{array}{c}28,3 \pm \\
0,4 \\
\end{array}$ & $\begin{array}{c}28,4^{+} \\
0,3 \\
\end{array}$ & $\begin{array}{c}28,4^{ \pm} \\
0,3 \\
\end{array}$ & $\begin{array}{c}28,1 \pm \\
0,3\end{array}$ \\
\hline \multirow{6}{*}{$20: 00$} & 0 & $\begin{array}{c}29,7 \pm \\
1,4 \\
\end{array}$ & $\begin{array}{c}30,0 \pm \\
0,7 \\
\end{array}$ & $\begin{array}{c}29,7 \pm \\
0,9 \\
\end{array}$ & $\begin{array}{c}29,8 \pm \\
1,0 \\
\end{array}$ & $\begin{array}{c}29,3 \pm \\
1,2\end{array}$ & $\begin{array}{c}29,0 \pm \\
1,2 \\
\end{array}$ & $\begin{array}{c}27,8 \pm \\
0,7 \\
\end{array}$ & $\begin{array}{c}27,8 \pm \\
0,8 \\
\end{array}$ & $\begin{array}{c}27,1 \pm \\
1,1 \\
\end{array}$ & $\begin{array}{c}27,4 \pm \\
0,9 \\
\end{array}$ & $\begin{array}{c}26,9 \pm \\
0,8 \\
\end{array}$ & $\begin{array}{c}26,5 \pm \\
0,5\end{array}$ \\
\hline & 5 & $\begin{array}{c}27,0 \pm \\
0,5 \\
\end{array}$ & $\begin{array}{c}27,2 \pm \\
0,4 \\
\end{array}$ & $\begin{array}{c}27,3 \pm \\
0,4 \\
\end{array}$ & $\begin{array}{c}27,9 \pm \\
0,7 \\
\end{array}$ & $\begin{array}{c}27,5 \pm \\
0,5 \\
\end{array}$ & $\begin{array}{c}27,4 \pm \\
0,4 \\
\end{array}$ & $\begin{array}{c}27,1 \pm \\
0,3 \\
\end{array}$ & $\begin{array}{c}27,4 \pm \\
0,4 \\
\end{array}$ & $\begin{array}{c}27,0 \pm \\
0,3 \\
\end{array}$ & $\begin{array}{c}27,0 \pm \\
0,4 \\
\end{array}$ & $\begin{array}{c}27,1 \pm \\
0,5 \\
\end{array}$ & $\begin{array}{c}26,5 \pm \\
0,2 \\
\end{array}$ \\
\hline & 10 & $\begin{array}{c}27,8 \pm \\
0,3 \\
\end{array}$ & $\begin{array}{c}27,9 \pm \\
0,4 \\
\end{array}$ & $\begin{array}{c}28,0 \pm \\
0,4 \\
\end{array}$ & $\begin{array}{c}28,6 \pm \\
0,7 \\
\end{array}$ & $\begin{array}{c}27,9 \pm \\
0,7\end{array}$ & $\begin{array}{c}27,9 \pm \\
0,4 \\
\end{array}$ & $\begin{array}{c}27,8 \pm \\
0,4 \\
\end{array}$ & $\begin{array}{c}27,9 \pm \\
0,5 \\
\end{array}$ & $\begin{array}{c}27,5 \pm \\
0,4 \\
\end{array}$ & $\begin{array}{c}27,5 \pm \\
0,4 \\
\end{array}$ & $\begin{array}{c}27,7 \pm \\
0,6 \\
\end{array}$ & $\begin{array}{c}27,0 \pm \\
0,3 \\
\end{array}$ \\
\hline & 20 & $\begin{array}{c}27,6 \pm \\
0,2 \\
\end{array}$ & $\begin{array}{c}27,9 \pm \\
0,4 \\
\end{array}$ & $\begin{array}{c}28,0 \pm \\
0,3 \\
\end{array}$ & $\begin{array}{c}28,5 \pm \\
0,4 \\
\end{array}$ & $\begin{array}{c}28,3 \pm \\
0,3 \\
\end{array}$ & $\begin{array}{c}28,2 \pm \\
0,4 \\
\end{array}$ & $\begin{array}{c}27,9 \pm \\
0,2 \\
\end{array}$ & $\begin{array}{c}27,9 \pm \\
0,3 \\
\end{array}$ & $\begin{array}{c}27,7^{ \pm} \\
0,3 \\
\end{array}$ & \begin{tabular}{|c|}
$27,8 \pm$ \\
0,3 \\
\end{tabular} & $\begin{array}{c}27,7 \pm \\
0,4 \\
\end{array}$ & $\begin{array}{c}27,3 \pm \\
0,2\end{array}$ \\
\hline & 30 & $\begin{array}{c}27,6 \pm \\
0,2 \\
\end{array}$ & $\begin{array}{c}27,7 \pm \\
0,7 \\
\end{array}$ & $\begin{array}{c}27,9 \pm \\
0,3 \\
\end{array}$ & $\begin{array}{c}28,4^{+} \\
0,3 \\
\end{array}$ & $\begin{array}{c}28,3 \pm \\
0,3 \\
\end{array}$ & $\begin{array}{c}28,2 \pm \\
0,3 \\
\end{array}$ & $\begin{array}{c}28,1 \pm \\
0,3 \\
\end{array}$ & $\begin{array}{c}28,0 \pm \\
0,2 \\
\end{array}$ & $\begin{array}{c}27,9 \pm \\
0,3 \\
\end{array}$ & $\begin{array}{c}28,0 \pm \\
0,2\end{array}$ & $\begin{array}{c}28,0 \pm \\
0,3 \\
\end{array}$ & $\begin{array}{c}27,5- \\
0,2 \\
\end{array}$ \\
\hline & 50 & $\begin{array}{c}27,9 \pm \\
0,2 \\
\end{array}$ & $\begin{array}{c}28,2 \pm \\
0,3\end{array}$ & $\begin{array}{c}28,1 \pm \\
0,3\end{array}$ & $\begin{array}{c}28,5^{ \pm} \\
0,3\end{array}$ & $\begin{array}{c}28,6 \pm \\
0,3\end{array}$ & $\begin{array}{c}28,5 \pm \\
0,3\end{array}$ & $\begin{array}{c}28,4 \pm \\
0,3\end{array}$ & $\begin{array}{c}28,2 \pm \\
0,4\end{array}$ & $\begin{array}{c}28,2 \pm \\
0,4\end{array}$ & $\begin{array}{c}28,2 \pm \\
0,3\end{array}$ & $\begin{array}{c}28,2 \pm \\
0,2\end{array}$ & $\begin{array}{c}28,0 \pm \\
0,2\end{array}$ \\
\hline
\end{tabular}

TABELA VI - Temperatura média de décadas, decênio 65-75, nas profundidades de 0,5, $10,20,30$ e $50 \mathrm{~cm}$. 


\begin{tabular}{|c|c|c|c|c|c|c|c|c|c|c|c|c|c|}
\hline \multirow{2}{*}{ HORAS } & \multirow[b]{2}{*}{$\begin{array}{c}\mathrm{z} \\
(\mathrm{cm}) \\
\end{array}$} & \multicolumn{12}{|c|}{$\frac{\text { DECENIO } 65-75}{\text { MESES }}$} \\
\hline & & JAN. & FEV. & MAR. & $\mathrm{ABR}$. & MAIO & JUN. & JUL. & AGO. & SET. & OUT. & NOV. & DEZ. \\
\hline \multirow{6}{*}{$8: 00$} & 0 & $\begin{array}{c}23,4 \pm \\
0,2 \\
\end{array}$ & $\begin{array}{c}23,4 \pm \\
0,2 \\
\end{array}$ & $\begin{array}{c}23,4 \pm \\
0,2\end{array}$ & $\begin{array}{c}23,6 \pm \\
0,2\end{array}$ & $\begin{array}{c}22,9 \pm \\
0,2\end{array}$ & $\begin{array}{c}23,1 \pm \\
0,2\end{array}$ & $\begin{array}{c}22,8 \pm \\
0,2\end{array}$ & $\begin{array}{c}23,0 \pm \\
0,2\end{array}$ & $\begin{array}{c}23,4 \pm \\
0,2\end{array}$ & $\begin{array}{c}23,7^{+} \\
0,2\end{array}$ & $\begin{array}{c}23,9 \pm \\
0,2\end{array}$ & $\begin{array}{c}23,6 \pm \\
0,2 \\
\end{array}$ \\
\hline & 5 & $\begin{array}{c}25,0 \pm \\
0,3 \\
\end{array}$ & $\begin{array}{c}25,0 \pm \\
0,2 \\
\end{array}$ & $\begin{array}{c}25,2 \pm \\
0,2 \\
\end{array}$ & $\begin{array}{c}25,4 \pm \\
0,2 \\
\end{array}$ & $\begin{array}{c}25,2 \pm \\
0,2 \\
\end{array}$ & $\begin{array}{c}25,1 \pm \\
0,2\end{array}$ & $\begin{array}{c}24,8 \pm \\
0,1 \\
\end{array}$ & $\begin{array}{c}25,1 \pm \\
0,2\end{array}$ & $\begin{array}{c}25,6 \pm \\
0,3 \\
\end{array}$ & $\begin{array}{c}26,1 \pm \\
0,2 \\
\end{array}$ & $\begin{array}{c}26,0 \pm \\
0,2\end{array}$ & $\begin{array}{c}25,7^{ \pm} \\
0,2 \\
\end{array}$ \\
\hline & 10 & $\begin{array}{c}25,5 \pm \\
0,2 \\
\end{array}$ & $\begin{array}{c}25,5 \pm \\
0,3 \\
\end{array}$ & $\begin{array}{c}25,6 \pm \\
0,2 \\
\end{array}$ & $\begin{array}{c}25,6 \pm \\
0,3 \\
\end{array}$ & $\begin{array}{c}25,3 \pm \\
0,2 \\
\end{array}$ & $\begin{array}{c}25,3^{+} \\
0,2 \\
\end{array}$ & $\begin{array}{c}25,2 \pm \\
0,1\end{array}$ & $\begin{array}{c}25,5 \pm \\
0,2\end{array}$ & $\begin{array}{c}26,1 \pm \\
0,2\end{array}$ & $\begin{array}{c}26,6 \pm \\
0,2\end{array}$ & $\begin{array}{c}26,3^{+} \\
0,2\end{array}$ & $\begin{array}{c}26,1 \pm \\
0,2\end{array}$ \\
\hline & 20 & $\begin{array}{c}26,3^{ \pm} \\
0,3 \\
\end{array}$ & $\begin{array}{c}26,3^{ \pm} \\
0,4 \\
\end{array}$ & $\begin{array}{c}26,6 \pm \\
0,2\end{array}$ & $\begin{array}{c}26,3 \pm \\
0,2 \\
\end{array}$ & $\begin{array}{c}26,2 \pm \\
0,2\end{array}$ & \begin{tabular}{|c|}
$26,2 \pm$ \\
0,1 \\
\end{tabular} & $\begin{array}{c}26,6 \pm \\
0,2 \\
\end{array}$ & $\begin{array}{c}27,1 \pm \\
0,1 \\
\end{array}$ & $\begin{array}{c}27,6 \pm \\
0,2\end{array}$ & $\begin{array}{c}27,3^{ \pm} \\
0,2 \\
\end{array}$ & $\begin{array}{c}27,0 \pm \\
0,2 \\
\end{array}$ & $\begin{array}{c}26,8 \pm \\
0,2 \\
\end{array}$ \\
\hline & 30 & $\begin{array}{c}27,0 \pm \\
0,2 \\
\end{array}$ & $\begin{array}{c}27,0 \pm \\
0,2 \\
\end{array}$ & $\begin{array}{c}27,1 \pm \\
0,2\end{array}$ & $\begin{array}{c}27,1 \pm \\
0,2 \\
\end{array}$ & $\begin{array}{c}26,8 \pm \\
0,2 \\
\end{array}$ & $\begin{array}{c}26,8 \pm \\
0,2 \\
\end{array}$ & $\begin{array}{c}26,7 \pm \\
0,3 \\
\end{array}$ & $\begin{array}{c}27,3^{ \pm} \\
0,2\end{array}$ & $\begin{array}{c}27,7 \pm \\
0,2 \\
\end{array}$ & $\begin{array}{c}28,3^{ \pm} \\
0,2 \\
\end{array}$ & $\begin{array}{c}27,9 \pm \\
0,2\end{array}$ & $\begin{array}{c}27,7 \pm \\
0,1 \\
\end{array}$ \\
\hline & 50 & $\begin{array}{c}27,7 \pm \\
0,2 \\
\end{array}$ & $\begin{array}{c}27,6 \pm \\
0,2 \\
\end{array}$ & $\begin{array}{c}27,6 \pm \\
0,2\end{array}$ & $\begin{array}{c}27,5 \pm \\
0,2 \\
\end{array}$ & $\begin{array}{c}27,2 \pm \\
0,2\end{array}$ & $\begin{array}{c}27,2 \pm \\
0,2\end{array}$ & $\begin{array}{c}27,1 \pm \\
0,2\end{array}$ & $\begin{array}{c}27,6 \pm \\
0,1 \\
\end{array}$ & $\begin{array}{c}28,1 \pm \\
0,2\end{array}$ & $\begin{array}{c}28,6 \pm \\
0,2\end{array}$ & $\begin{array}{c}28,3 \pm \\
0,2\end{array}$ & $\begin{array}{c}28,1 \pm \\
0,2\end{array}$ \\
\hline \multirow{6}{*}{$14: 00$} & 0 & $\begin{array}{c}31,2 \pm \\
0,6 \\
\end{array}$ & $\begin{array}{c}31,1 \pm \\
0,8 \\
\end{array}$ & $\begin{array}{c}31,6 \pm \\
0,7\end{array}$ & $\begin{array}{c}31,5 \pm \\
0,6 \\
\end{array}$ & $\begin{array}{c}31,8 \pm \\
0,7\end{array}$ & $\begin{array}{c}32,6 \pm \\
0,9 \\
\end{array}$ & $\begin{array}{c}33,5 \pm \\
0,8\end{array}$ & $\begin{array}{c}35,5 \pm \\
0,7 \\
\end{array}$ & $\begin{array}{c}36,0 \pm \\
0,9\end{array}$ & $\begin{array}{c}36,0 \pm \\
0,8 \\
\end{array}$ & $\begin{array}{c}33,3 \pm \\
0,6\end{array}$ & $\begin{array}{c}32,4 \pm \\
0,7\end{array}$ \\
\hline & 5 & $\begin{array}{c}28,1 \pm \\
0,3 \\
\end{array}$ & $\begin{array}{c}28,2 \pm \\
0,3 \\
\end{array}$ & $\begin{array}{c}28,6 \pm \\
0,5\end{array}$ & $\begin{array}{c}28,7^{ \pm} \\
0,3 \\
\end{array}$ & $\begin{array}{c}28,4^{ \pm} \\
0,3 \\
\end{array}$ & $\begin{array}{c}28,6^{ \pm} \\
0,3 \\
\end{array}$ & $\begin{array}{c}28,8 \pm \\
0,4 \\
\end{array}$ & $\begin{array}{c}28,7 \pm \\
0,4 \\
\end{array}$ & $\begin{array}{c}30,0 \pm \\
0,4 \\
\end{array}$ & $\begin{array}{c}30,3 \pm \\
0,5\end{array}$ & $\begin{array}{c}29,1 \pm \\
0,3 \\
\end{array}$ & $\begin{array}{c}28,6 \pm \\
0,4 \\
\end{array}$ \\
\hline & 10 & $\begin{array}{c}27,6 \pm \\
0,2 \\
\end{array}$ & $\begin{array}{c}27,8 \pm \\
0,4 \\
\end{array}$ & $\begin{array}{c}27,8 \pm \\
0,3 \\
\end{array}$ & $\begin{array}{c}28,0 \pm \\
0,3 \\
\end{array}$ & $\begin{array}{c}27,7 \pm \\
0,2 \\
\end{array}$ & $\begin{array}{c}27,8 \pm \\
0,2 \\
\end{array}$ & $\begin{array}{c}27,8 \pm \\
0,3 \\
\end{array}$ & $\begin{array}{c}28,6 \pm \\
0,2 \\
\end{array}$ & $\begin{array}{c}29,1 \pm \\
0,4 \\
\end{array}$ & $\begin{array}{c}29,1 \pm \\
0,3 \\
\end{array}$ & $\begin{array}{c}28,4^{+} \\
0,2\end{array}$ & $\begin{array}{c}28,1 \pm \\
0,2 \\
\end{array}$ \\
\hline & 20 & $\begin{array}{c}27,4 \pm \\
0,2 \\
\end{array}$ & $\begin{array}{c}27,4 \pm \\
0,2 \\
\end{array}$ & $\begin{array}{c}27,4 \pm \\
0,3 \\
\end{array}$ & $\begin{array}{c}27,4 \pm \\
0,2 \\
\end{array}$ & $\begin{array}{c}27,3^{+} \\
0,2 \\
\end{array}$ & $\begin{array}{c}27,1 \pm \\
0,2 \\
\end{array}$ & $\begin{array}{c}27,1 \pm \\
0,1 \\
\end{array}$ & $\begin{array}{c}27,8 \pm \\
0,2 \\
\end{array}$ & $\begin{array}{c}28,2 \pm \\
0,2 \\
\end{array}$ & $\begin{array}{c}28,7 \pm \\
0,2 \\
\end{array}$ & $\begin{array}{c}28,2 \pm \\
0,1 \\
\end{array}$ & $\begin{array}{c}27,9 \pm \\
0,2 \\
\end{array}$ \\
\hline & 30 & $\begin{array}{c}27,4 \pm \\
0,2 \\
\end{array}$ & $\begin{array}{c}27,3 \pm \\
0,2 \\
\end{array}$ & $\begin{array}{c}27,3^{+} \\
0,2 \\
\end{array}$ & $\begin{array}{c}27,3^{+} \\
0,2 \\
\end{array}$ & $\begin{array}{c}27,0 \pm \\
0,2 \\
\end{array}$ & $\begin{array}{c}27,0 \pm \\
0,2 \\
\end{array}$ & $\begin{array}{c}26,9 \pm \\
0,1 \\
\end{array}$ & $\begin{array}{c}27,5 \pm \\
0,1 \\
\end{array}$ & $\begin{array}{c}27,9 \pm \\
0,2 \\
\end{array}$ & $\begin{array}{c}28,5 \pm \\
0,2 \\
\end{array}$ & $\begin{array}{c}28,1 \pm \\
0,1 \\
\end{array}$ & $\begin{array}{c}27,9 \pm \\
0,1 \\
\end{array}$ \\
\hline & 50 & $\begin{array}{c}27,8 \pm \\
0,1 \\
\end{array}$ & $\begin{array}{c}27,7^{+} \\
0,2 \\
\end{array}$ & $\begin{array}{c}27,7^{+} \\
0,2 \\
\end{array}$ & $\begin{array}{c}27,6 \pm \\
0,2 \\
\end{array}$ & $\begin{array}{c}27,3 \pm \\
0,2\end{array}$ & \begin{tabular}{|c|}
$27,3^{+}$ \\
0,2 \\
\end{tabular} & $\begin{array}{c}27,2 \pm \\
0,1\end{array}$ & \begin{tabular}{|c|}
$27,7 \pm$ \\
0,2 \\
\end{tabular} & $\begin{array}{c}28,2 \pm \\
0,1 \\
\end{array}$ & $\begin{array}{c}28,7 \pm \\
0,1 \\
\end{array}$ & $\begin{array}{c}28,4 \pm \\
0,2 \\
\end{array}$ & $\begin{array}{c}28,3^{ \pm} \\
0,1 \\
\end{array}$ \\
\hline \multirow{6}{*}{$20: 00$} & 0 & $\begin{array}{c}25,7^{ \pm} \\
0,3 \\
\end{array}$ & $\begin{array}{c}25,7^{ \pm} \\
0,3 \\
\end{array}$ & $\begin{array}{c}26,0 \pm \\
0,4\end{array}$ & $\begin{array}{c}26,4^{+} \\
0,3 \\
\end{array}$ & $\begin{array}{c}26,9 \pm \\
0,5 \\
\end{array}$ & $\begin{array}{c}27,6 \pm \\
0,6 \\
\end{array}$ & $\begin{array}{c}27,8 \pm \\
0,5 \\
\end{array}$ & \begin{tabular}{|c|}
$29,5 \pm$ \\
0,6 \\
\end{tabular} & $\begin{array}{c}29,8 \pm \\
0,5 \\
\end{array}$ & $\begin{array}{c}29,4 \pm \\
0,6 \\
\end{array}$ & $\begin{array}{c}27,6 \pm \\
0,4\end{array}$ & $\begin{array}{c}26,8 \pm \\
0,4 \\
\end{array}$ \\
\hline & 5 & $\begin{array}{c}26,5 \pm \\
0,2 \\
\end{array}$ & $\begin{array}{c}26,3 \pm \\
0,3 \\
\end{array}$ & $\begin{array}{c}26,5 \pm \\
0,2 \\
\end{array}$ & $\begin{array}{c}26,4 \pm \\
0,2 \\
\end{array}$ & $\begin{array}{c}26,3 \pm \\
0,2\end{array}$ & $\begin{array}{c}26,3^{ \pm} \\
0,2 \\
\end{array}$ & $\begin{array}{c}26,3 \pm \\
0,2 \\
\end{array}$ & $\begin{array}{c}26,8 \pm \\
0,2 \\
\end{array}$ & $\begin{array}{c}27,2 \pm \\
0,2 \\
\end{array}$ & $\begin{array}{c}27,6 \pm \\
0,3 \\
\end{array}$ & $\begin{array}{c}27,2 \pm \\
0,2\end{array}$ & $\begin{array}{c}26,8 \pm \\
0,2 \\
\end{array}$ \\
\hline & 10 & $\begin{array}{c}27,0 \pm \\
0,2 \\
\end{array}$ & $\begin{array}{c}27,0 \pm \\
0,3 \\
\end{array}$ & $\begin{array}{c}27,1 \pm \\
0,2\end{array}$ & $\begin{array}{c}27,0 \pm \\
0,2 \\
\end{array}$ & $\begin{array}{c}26,8 \pm \\
0,3 \\
\end{array}$ & $\begin{array}{c}26,8 \pm \\
0,2\end{array}$ & $\begin{array}{c}26,7 \pm \\
0,2 \\
\end{array}$ & $\begin{array}{c}27,4 \pm \\
0,2 \\
\end{array}$ & $\begin{array}{c}27,9 \pm \\
0,2 \\
\end{array}$ & $\begin{array}{c}28,2 \pm \\
0,3 \\
\end{array}$ & $\begin{array}{c}28,1 \pm \\
0,7\end{array}$ & $\begin{array}{c}27,4 \pm \\
0,3 \\
\end{array}$ \\
\hline & 20 & $\begin{array}{c}27,0 \pm \\
0,2 \\
\end{array}$ & $\begin{array}{c}27,0 \pm \\
0,2 \\
\end{array}$ & $\begin{array}{c}27,1 \pm \\
0,2 \\
\end{array}$ & $\begin{array}{c}27,0 \pm \\
0,2 \\
\end{array}$ & $\begin{array}{c}26,8 \pm \\
0,2 \\
\end{array}$ & \begin{tabular}{|c|}
$26,8 \pm$ \\
0,2 \\
\end{tabular} & $\begin{array}{c}26,7 \pm \\
0,2 \\
\end{array}$ & $\begin{array}{c}27,4 \pm \\
0,1 \\
\end{array}$ & $\begin{array}{c}27,8 \pm \\
0,2 \\
\end{array}$ & $\begin{array}{c}28,3 \pm \\
0,2\end{array}$ & $\begin{array}{c}27,8 \pm \\
0,2 \\
\end{array}$ & $\begin{array}{c}27,6 \pm \\
0,2 \\
\end{array}$ \\
\hline & 30 & $\begin{array}{c}27,2 \pm \\
0,2 \\
\end{array}$ & $\begin{array}{c}27,2 \pm \\
0,2 \\
\end{array}$ & $\begin{array}{c}27,2 \pm \\
0,2\end{array}$ & $\begin{array}{c}27,1 \pm \\
0,2 \\
\end{array}$ & $\begin{array}{c}26,8 \pm \\
0,2 \\
\end{array}$ & $\begin{array}{c}27,0 \pm \\
0,1 \\
\end{array}$ & $\begin{array}{c}26,7 \pm \\
0,2\end{array}$ & $\begin{array}{c}27,4 \pm \\
0,1 \\
\end{array}$ & $\begin{array}{c}27,7 \pm \\
0,2 \\
\end{array}$ & $\begin{array}{c}28,3 \pm \\
0,2 \\
\end{array}$ & $\begin{array}{c}28,0 \pm \\
0,2 \\
\end{array}$ & $\begin{array}{c}27,7 \pm \\
0,2 \\
\end{array}$ \\
\hline & 50 & $\begin{array}{c}27,7 \pm \\
0,1 \\
\end{array}$ & $\begin{array}{c}27,6 \pm \\
0,2\end{array}$ & $\begin{array}{c}27,6 \pm \\
0,2\end{array}$ & $\begin{array}{c}27,4^{ \pm} \\
0,2 \\
\end{array}$ & $\begin{array}{c}27,1 \pm \\
0,2\end{array}$ & $\begin{array}{c}27,2 \pm \\
0,2\end{array}$ & $\begin{array}{c}27,1 \pm \\
0,1\end{array}$ & $\begin{array}{c}27,6 \pm \\
0,3 \\
\end{array}$ & $\begin{array}{c}28,1 \pm \\
0,1\end{array}$ & $\begin{array}{c}28,5 \pm \\
0,2\end{array}$ & $\begin{array}{c}28,3 \pm \\
0,2\end{array}$ & $\begin{array}{c}28,2 \pm \\
0,2\end{array}$ \\
\hline
\end{tabular}

TABELA VII - Temperatura média mensal, decênio $65-75$, nas profundidades de $0,5,10$, 20,30 e $50 \mathrm{~cm},{ }^{\circ} \mathrm{C}$. 


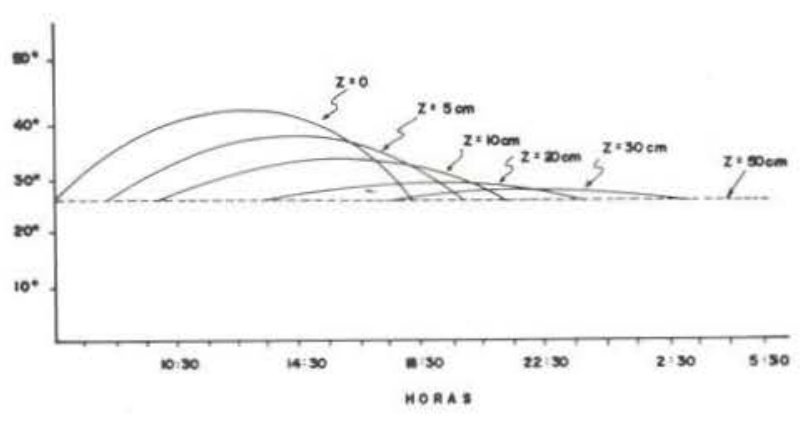

Gráfico II. Variação diária (período diurno) da temperatura em seis profundidades (média do mês de setembro, década 65-75).

A variação anual das temperaturas para os três horários está representada no gráfico III, onde pode-se visualizar a variabilidade no regime térmico do solo em $\mathrm{Z}=0$.

\section{CAMADA DE EXTINÇÄO}

Sendo que a difusividade térmica é praticamente constante para um determinado solo, a espessura da camada de extinção para a onda diária da temperatura fica sendo uma função da amplitude $\left(T_{0}\right)$ e da diferença $T_{\text {max }}-\bar{T}$. A diferença $T_{\text {max }}-\bar{T}$ é eleita de acordo com o fenômeno a ser estudado e em função da precisão dos instrumentos de medida. Neste caso particular admitiu-se que a camada de extinção atinge profundidades onde se fazem sentir flutuações de temperatura da ordem de grandeza de $0,1^{\circ} \mathrm{C}$, conforme Tabela II.

\section{ABSORÇÃo DE CALOR PELO SOLO}

Com os dados disponíveis do actinógrafo, verificou-se que a quantidade de energia radiante global que atinge a superfície da Terra é da ordem de $423 \mathrm{cal} \mathrm{cm}^{-2} \mathrm{dia}^{-1}$ (os dados disponíveis são os do ano de 1976, o que nos limita de tirar maiores informações). Analisando a Tabela III vê-se que a energia absorvida pelo solo do nascer ao pôr do sol é da ordem de $95 \mathrm{cal} \mathrm{cm}^{-2}$, ou em outras palavras, o solo absorve $22 \%$ da energia incidente naquelas condições de cobertura, o que concorda muito bem com os dados existentes na literatura.

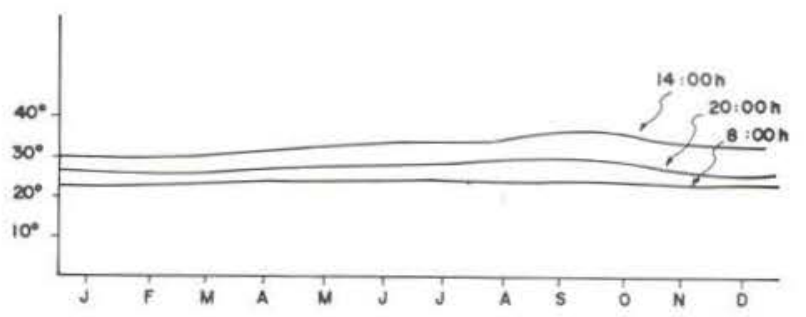

Gráfico III. Variação anual da temperatura media da década $65-75$, para os três horários de observação.

\section{CoNCLUSÕES}

1. A temperatura do solo na superfície às 8:00 horas é de $23,4^{\circ} \mathrm{C}$ e é constante para o ano todo.

\begin{tabular}{|c|c|c|c|c|c|c|c|c|c|c|c|c|}
\hline Meses & Jan. & Fev. & Mar. & Abril & Maio & Jun. & Jul. & Ago. & Set. & Out. & Nov. & Dez. \\
\hline $\mathbf{z}$ & 59,10 & 59,10 & 59,50 & 59,70 & 59,60 & 59,80 & 60,10 & 59,70 & 60,70 & 60,60 & 59,60 & 59,10 \\
\hline
\end{tabular}

TABELA II - Espessura da camada de extinção, média mensal expressa em cm.

\begin{tabular}{c|c|c|c|c|c|c|c|c|c|c|c|c}
\hline Meses & Jan. & Fev. & Mar. & Abril & Maio & Jun. & Jul. & Ago. & Set. & Out. & Nov. & Dez. \\
\hline $\mathbf{F}$ & 90,50 & 89,10 & 92,60 & 94,30 & 93,40 & 95,10 & 97,50 & 93,70 & 102,30 & 102,00 & 93,20 & 84,40 \\
\hline
\end{tabular}

TABELA III - Calor absorvido pelo solo do nascer ao pôr do sol, média mensal em cal/cm? 
2. A temperatura máxima da superfície do solo $(Z=O)$ nos meses de setembro, outubro e novembro é de aproximadamente $46^{\circ} \mathrm{C}$, enquanto que nos demais meses ela é de $43,5^{\circ} \mathrm{C}$.

3. Para se estudar fenômenos que possuam sensibilidade de variação de temperatura maior que $0,1^{\circ} \mathrm{C}$, a camada de solo a considerar é de aproximadamente $60 \mathrm{~cm}$.

4. Os solos da região estudada e com cobertura de grama rasteira absorve, do nascer ao pôr do sol, em média $95 \mathrm{cal}$ $\mathrm{cm}^{-2}$, o que representa em média $22 \%$ da radiação solar global.

\section{AgRADECIMENTOS}

Os autores agradecem ao Dr. W. L. F. Brinkmann por ter iniciado a obtenção dos dados meteorológicos.

\section{SUMMARX}

With a view to providing information for a complete micrometeorological study of the Reserva Florestal Ducke (Ducke Forest Reserve), some thermal soil parameters have been estimated, under conditions of soil grown with short grass. The estimated data indicate that during the months of September, October and November the soil surface temperature reaches its highest values, of the order of $46^{\circ} \mathrm{C}$, while during the remaining months of the year it reaches values around $43.5^{\circ} \mathrm{C}$. The heat absorbed by the soil is of the order of $95 \mathrm{cal}$ $\mathrm{cm}^{-2}$ day -1 , corresponding to $22 \%$ of the incident global energy. The daily temperature wave disappears in a soil surface layer which is approximately $60 \mathrm{~cm}$ thick.

\section{BIBLIOGRAFIA CITADA}

Decico, A.

1974 - Determinação das propriedades térmi. cas do solo. Tese de Livre-Docência. Piracicaba, SP., Universidade de São Paulo. 